\title{
How Method-Dependent Are Calculated Differences between Vertical, Adiabatic, and 0- 0 Excitation Energies?
}

Changfeng Fang, Baswanth Oruganti and Bo Durbeej

\section{Linköping University Post Print}

\section{Tweet}

N.B.: When citing this work, cite the original article.

Original Publication:

Changfeng Fang, Baswanth Oruganti and Bo Durbeej, How Method-Dependent Are Calculated Differences between Vertical, Adiabatic, and 0-0 Excitation Energies?, 2014, Journal of Physical Chemistry A, (118), 23, 4157-4171.

http://dx.doi.org/10.1021/jp501974p

Copyright: American Chemical Society http://pubs.acs.org/

Postprint available at: Linköping University Electronic Press http://urn.kb.se/resolve?urn=urn:nbn:se:liu:diva-109132 


\section{How Method-Dependent Are Calculated Differences Between Vertical, Adiabatic and 0-0 Excitation Energies?}

Changfeng Fang, Baswanth Oruganti and Bo Durbeej*

Division of Computational Physics, IFM, Linköping University, SE-581 83 Linköping, Sweden 


\section{ABSTRACT:}

Through a large number of benchmark studies, the performance of different quantum chemical methods in calculating vertical excitation energies is today quite well established. Furthermore, these efforts have in recent years been complemented by a few benchmarks focusing instead on adiabatic excitation energies. However, it is much less well established how calculated differences between vertical, adiabatic and 0-0 excitation energies vary between methods, which may be due to the cost of evaluating zero-point vibrational energy corrections for excited states. To fill this gap, we have calculated vertical, adiabatic and 0-0 excitation energies for a benchmark set of molecules covering both organic and inorganic systems. Considering in total 96 excited states and using both TD-DFT with a variety of exchange-correlation functionals and the ab initio CIS and $\mathrm{CC} 2$ methods, it is found that while the vertical excitation energies obtained with the various methods show an average (over the 96 states) standard deviation of $0.39 \mathrm{eV}$, the corresponding standard deviations for the differences between vertical, adiabatic and 0-0 excitation energies are much smaller: 0.10 (difference between adiabatic and vertical) and $0.02 \mathrm{eV}$ (difference between $0-0$ and adiabatic). These results provide a quantitative measure showing that the calculation of such quantities in photochemical modeling is well amenable to low-level methods. In addition, we also report on how these energy differences vary between chemical systems and assess the performance of TD-DFT, CIS and $\mathrm{CC} 2$ in reproducing experimental 0-0 excitation energies.

\section{KEYWORDS:}

- Excited states $\bullet$ TD-DFT $\bullet$ Ab initio $\bullet$ Vibrational corrections $\bullet$ Benchmark 


\section{INTRODUCTION}

Through a number of benchmark studies, it is today rather well established how different quantum chemical methods perform in calculating electronically excited states of molecular systems. ${ }^{1-15}$ Accordingly, there are high-level ab initio methods capable of accurately describing various types of excited states for a range of chemical systems, such as complete active space second-order perturbation theory (CASPT2) ${ }^{16,17}$ and methods based on coupled cluster theory, ${ }^{18-20}$ but also methods rooted in time-dependent density functional theory (TD-DFT) ${ }^{21-27}$ that exhibit a more favorable cost-performance ratio. Thus, it is not surprising that TD-DFT, which for certain problems performs similarly to advanced ab initio approaches, is currently the most widely used tool for modeling excited states of medium-sized and large molecules, although the methodology is less broadly applicable than, e.g., CASPT2. For example, conventional TD-DFT is best suited for states dominated by single excitations. ${ }^{28}$

In most cases, benchmarks of excited-state methods focus on vertical excitation energies and use as reference data either experimental absorption maxima or vertical excitation energies obtained with high-level ab initio methods. While this procedure has been instrumental in forming a foundation for evaluating how well common methods in the field describe different types of excited states, it rests on three key assumptions. First, it is assumed that the electronic transition occurs without changes in the positions of the nuclei from their ground-state configuration (the Franck-Condon principle). Second, it is assumed that neither vibrational nor rotational effects influence the experimental 
absorption maxima. Third, when reference data are taken from high-level calculations, computational errors are thought to be small.

In the last few years, many different directions have been taken to go beyond the standard procedure for benchmarking quantum chemical methods for applications to excited states, or to investigate the potential pitfalls that this procedure entails. For example, rather than focusing on vertical excitation energies, Furche and co-workers ${ }^{29}$ compiled a large set of adiabatic excitation energies from high-resolution gas-phase experiments, and tested how well these energies are reproduced by TD-DFT and two correlated ab initio approaches: the approximate coupled-cluster singles and doubles $(\mathrm{CC} 2)^{30}$ method and the second-order algebraic diagrammatic construction approximation $(\mathrm{ADC}(2))^{31,32}$ method. Accordingly, by accounting for excited-state relaxation by means of analytic gradient techniques, this study considered energy differences between ground and excited states at their respective equilibrium geometries. Although much more expensive than ground-state geometry optimizations, such calculations are today feasible for many excited-state methods, including TD-DFT. ${ }^{33-48}$

Adding a further dimension to efforts along those lines and of relevance for determining which type of transition (vertical or adiabatic) best corresponds to experimental absorption maxima, are simulations of vibrationally resolved electronic absorption spectra reported by a number of research groups ${ }^{49-53}$ Such simulations require computation of vibrational wavefunctions and their overlap (Franck-Condon factors), and may also include a dependence of the electronic dipole moment operator on nuclear coordinates (Herzberg-Teller corrections). 
Focusing instead on improving the quality of available reference data for excitedstate studies, Thiel and co-workers ${ }^{7}$ assembled a benchmark set of best estimates of vertical excitation energies for 28 medium-sized organic molecules covering many important classes of chromophores. This was done by performing calculations with CASPT2 and a hierarchy of coupled cluster methods, as well as by extensively surveying the literature for correlated ab initio results obtained with large basis sets.

Despite the above-cited benchmark studies assessing the accuracy of ab initio and TD-DFT methods for calculating vertical excitation energies ${ }^{1-15}$ and a few such studies considering instead adiabatic transitions, ${ }^{29,41,54-58}$ the important issue of establishing the variation between methods in their estimates of the difference between vertical and adiabatic excitation energies has not been addressed in greater detail. Following a preliminary investigation along those lines that focused on the chromophores of the photoactive yellow protein and the green fluorescent protein, ${ }^{59}$ this study attempts to meet this objective in a much more comprehensive fashion. Specifically, using both TDDFT and ab initio methods, we calculate vertical and zero-point vibrational energy (ZPVE)-corrected adiabatic excitation energies for most of the molecules and states contained in the large (91 molecules and 109 adiabatic excitation energies) set of reference data collected by Furche and co-workers, ${ }^{29}$ and evaluate how the differences between these energies vary with the choice of method. Besides providing valuable insight into how method-sensitive computed excited-state relaxation energies are, such information helps quantifying how large an error might be introduced when a computational analysis of an experimental absorption spectrum is based on vertical excitation energies alone. 
It is clear that by not primarily focusing on the accuracy with which excited-state methods reproduce adiabatic excitation energies, this work takes a different angle from previous works in the field. Nonetheless, it is important at this stage to point out the key differences, in terms of chemical systems studied and methods employed, relative to two of the most extensive benchmarks for the computation of adiabatic excitation energies already reported in the literature.

First, with regard to the work by Furche and co-workers, ${ }^{29}$ we consider a slightly reduced version of the 109-member set of excited states introduced by them. However, whereas their study was based exclusively on geometries and vibrational frequencies computed with the B3LYP ${ }^{60-62}$ hybrid density functional and invoked other functionals (LSDA, ${ }^{63} \mathrm{BP} 6,{ }^{64,65} \mathrm{PBE},{ }^{66} \mathrm{TPSS}^{67}$ and PBE0 ${ }^{68}$ ) for singlepoint energy calculations only, we perform all requisite calculations to obtain vertical and adiabatic excitation energies for the current 96 states with an alternative set of functionals (BP86, B3LYP, PBE0, M06-2X, ${ }^{10}$ M06-HF, ${ }^{69}$ CAM-B3LYP ${ }^{70}$ and $\omega B$ B7X-D ${ }^{71,72}$ ). Thereby, we are able to account for more recent progress in the development of improved density functionals, including efforts aimed directly at studies of excited states (e.g., M06-HF and CAMB3LYP). ${ }^{69,70}$ Furthermore, whereas Furche and co-workers ${ }^{29}$ for a smaller 15-member subset of states performed singlepoint energy calculations with the ab initio $\mathrm{CC} 2^{30}$ and $\operatorname{ADC}(2)^{31,32}$ methods, we use the CC2 method on equal footing with the DFT-based methods for all of the current 96 states. In other words, we employ CC2 also for geometry optimizations and frequency calculations.

Second, with regard to the work by Jacquemin and co-workers, ${ }^{57}$ who looked at a 40-member set of excited states of large conjugated dyes in solution and focused on the 
performance of TD-DFT, our 96-member set derives from smaller molecules, pertains to the gas phase and thereby enables testing the accuracy of the methods without introducing errors from the treatment of solvent effects, shows greater chemical variation among the constituting molecules, and, as noted above, is subjected also to CC2 calculations.

\section{METHODOLOGY}

2.1. Composition of benchmark set. The 96-member set of excited states for which vertical and adiabatic excitation energies were calculated cover all of the singlet and triplet states in the 109-member set previously compiled from high-resolution gas-phase

experiments. ${ }^{29}$ While that set also includes a few doublet states of organic and inorganic radicals and higher spin-multiplicity states of transition-metal compounds, such states oftentimes require more elaborate methods than TD-DFT and CC2 that explicitly account for multi-reference correlation effects, ${ }^{73}$ and were therefore not considered in this work. With a few exceptions, the singlet and triplet states subjected to calculations are the lowest excited states $\left(\mathrm{S}_{1}\right.$ and $\left.\mathrm{T}_{1}\right)$ of these spin multiplicities.

As detailed in Table 1, the states cover a range of 79 different molecules that can be classified into nine different groups. Represented among these molecules are common classes of organic chromophores such as polyenes, carbonyl compounds, aromatic hydrocarbons and heterocyclic aromatic compounds, but also a number of small inorganic main-group and transition-metal compounds. Most of the states are of valence type and are dominated by a one-electron excitation from a single-reference ground state, 
and are hence particularly well suited for TD-DFT and CC2 calculations. Furthermore,

none of the states has charge-transfer character.

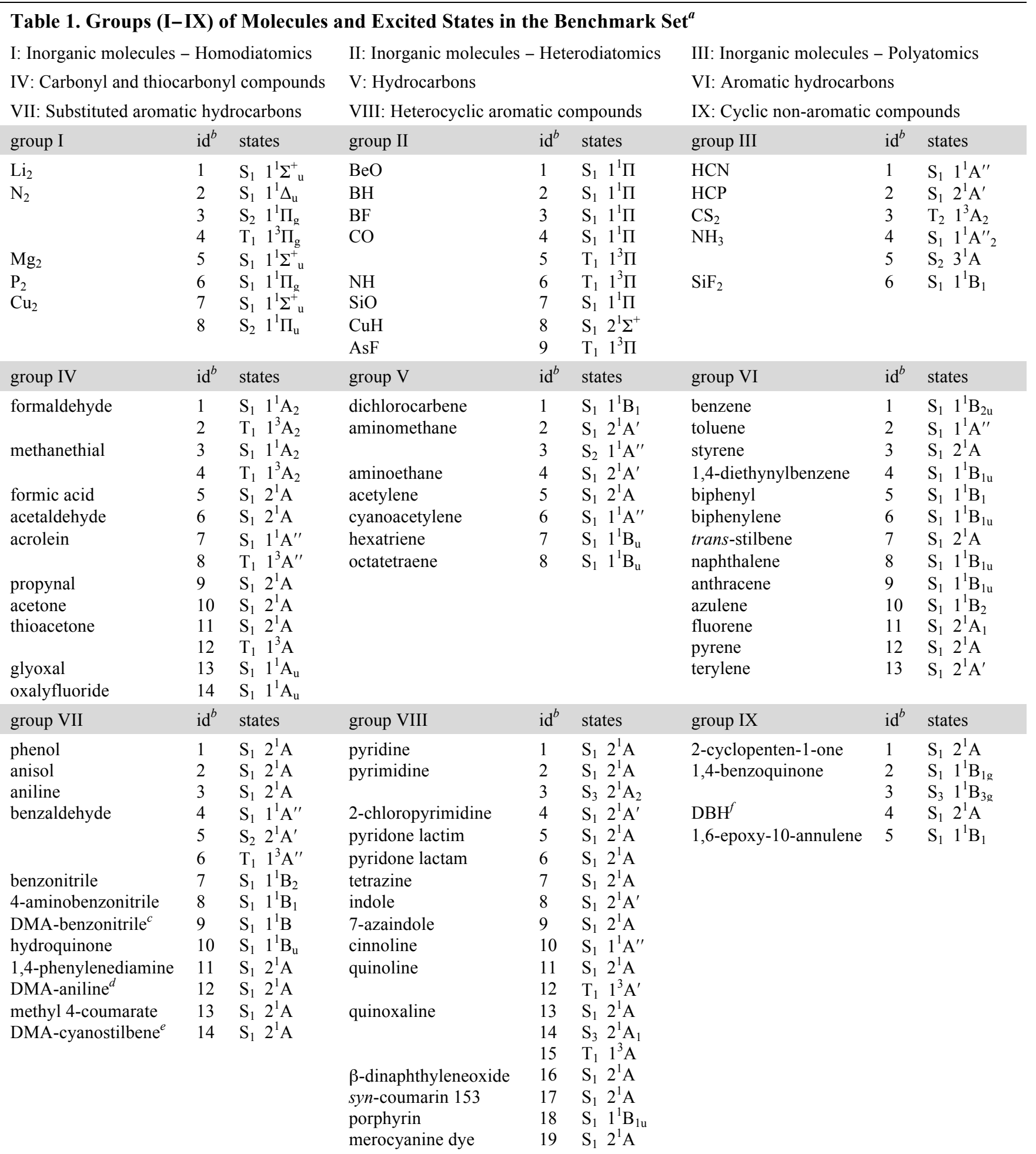


${ }^{a}$ The molecules in groups IV-IX are depicted in Figure S1 of the Supporting Information. Symmetry labels reflect molecular geometries after excited-state relaxation. ${ }^{b}$ Label used in figures. ${ }^{c} 4$-(dimethylamino)benzonitrile. ${ }^{d} 4$-(dimethylamino)aniline. ${ }^{e} 4$-(dimethylamino)-4'cyanostilbene. ${ }^{f}$ 2,3-diazabicyclo[2.2.1]-hept-2-ene.

2.2. Computational details. All ground-state DFT and excited-state TD-DFT calculations were performed using seven different density functionals (BP86, ${ }^{64,65}$ B3LYP ${ }^{60-62}$ PBE0,${ }^{68}$ M06-2X, ${ }^{10}$ M06-HF, ${ }^{69} \mathrm{CAM}^{6} \mathrm{BLYP}{ }^{70}$ and $\omega \mathrm{B} 97 \mathrm{X}-\mathrm{D}^{71,72}$ ) in combination with Dunning's correlation-consistent polarized valence double- $\zeta$ (ccpVDZ) basis set. ${ }^{74}$ Similarly, all CC2 calculations were done with an auxiliary cc-pVDZ basis $\operatorname{set}^{75}$ within the resolution-of-the-identity approximation. Of the seven density functionals employed, all are based on the generalized gradient approximation (GGA) and have shown good performance in many TD-DFT benchmarks. ${ }^{6,8-10,12-15}$ However, for a large set of excited states like the present one, there will inevitably be cases where the character of a particular state is such that it would have been worthwhile to consider also alternative functionals.

Briefly, BP86 is a "pure" GGA that does not introduce any exact, Hartree-Fock (HF) exchange in the exchange-correlation potential; B3LYP and PBE0 are "global" hybrid GGAs that contain a fixed fraction (20 and $25 \%$, respectively) of exact exchange; M06-2X (54 \%) and M06-HF (100\%) are global hybrid meta-GGAs that also include a dependence on the kinetic energy density; and CAM-B3LYP and $\omega$ B97X-D are longrange-corrected hybrid GGAs that allow the fraction of exact exchange to vary with the interelectronic distance - small at short range and larger at long range. Of these methods, some have been developed specifically with an eye toward TD-DFT applications. For example, owing to the inclusion of full exact exchange, M06-HF has been found to give a 
good description of excited states that have Rydberg character, ${ }^{10,69}$ which for many functionals pose a much greater challenge than valence states. ${ }^{23,76,77}$ Furthermore, through a partitioning of the electron repulsion operator into short-range (modeled with an exchange functional) and long-range (modeled with HF exchange) parts by means of the standard error function, ${ }^{78,79}$ CAM-B3LYP and $\omega$ B97X-D have shown good performance for long-range charge-transfer excited states, ${ }^{70-72}$ which are difficult to describe properly with standard functionals. ${ }^{3,26}$ Although, as noted above, no such states are included in this work, it is nonetheless of interest to assess how CAM-B3LYP and $\omega B$ 97X-D differ from other functionals also when applied to valence states.

First, ground-state geometries were optimized using DFT and CC2, where the latter optimizations were carried out with Hättig's implementation of analytic CC2 gradients. ${ }^{80}$ Based on the resulting geometries, vertical excitation energies were then obtained by performing TD-DFT and CC2 singlepoint calculations, where BP86 was used for $\mathrm{BP} 86$ geometries, $\mathrm{CC} 2$ for $\mathrm{CC} 2$ geometries, etc. In this way, rather than using a common set of (e.g., B3LYP) geometries for all singlepoint calculations, the observed differences between the estimates of the vertical excitation energies reflect also that the methods yield different equilibrium geometries. While it certainly would have been possible to use a larger basis set than cc-pVDZ for the singlepoint calculations, this option was not explored since we are here focusing more on differences between computational methods than on achieving the best possible agreement with experimental data. Furthermore, for most of the current excited states, expanding the basis set from double- $\zeta$ to triple- $\zeta$ quality, or including diffuse basis functions, has a marginal effect on the excitation energies obtained with TD-DFT. ${ }^{29}$ 
Second, excited-state geometries were optimized using analytic TD-DFT ${ }^{45-47}$ and $\mathrm{CC}_{2}{ }^{43}$ excited-state gradients. Then, adiabatic excitation energies were obtained as electronic energy differences between ground and excited states at their respective equilibrium geometries, to which were subsequently added ZPVE corrections derived from ground and excited-state frequency calculations at optimized ground and excitedstate structures, respectively. The frequency calculations were throughout performed at the same level of theory as the preceding geometry optimizations, and identified all geometries as potential energy minima with real vibrational frequencies only. While all ground-state DFT frequencies were determined by analytic second-derivative methods, the CC2 and TD-DFT frequencies were obtained through numerical differentiation of analytic gradients using finite differences, which by far constitutes the most resourcedemanding part of this work. Recently, and for the benefit of future studies in the field, an analytic approach for computing TD-DFT frequencies has been reported by Liu and Liang. ${ }^{81}$

In addition to using TD-DFT and $\mathrm{CC} 2$, vertical and adiabatic excitation energies were also calculated with the configuration interaction singles (CIS) method. ${ }^{34}$ In line with the overall strategy that every estimate of an excitation energy at a particular level of theory should be based on calculations performed at that very level, the requisite groundstate geometries for these calculations were optimized with the HF method. Both groundstate HF and excited-state CIS frequencies were determined using analytic secondderivative methods.

All calculations were carried out with the GAUSSIAN $09^{82}$ and TURBOMOLE $6.3^{83,84}$ (for CC2 calculations with the RICC2 module ${ }^{85}$ ) suites of programs. 


\section{RESULTS AND DISCUSSION}

In what follows, and as illustrated in Figure 1, vertical excitation energies, purely electronic adiabatic excitation energies, and ZPVE-corrected adiabatic excitation energies (or 0-0 excitation energies) are denoted $\Delta E_{\mathrm{ve}}, \Delta E_{\mathrm{ad}}$, and $\Delta E_{00}$, respectively. Furthermore, $\Delta \Delta E_{\text {ad }}=\Delta E_{\text {ad }}-\Delta E_{\text {ve }}$ (a non-positive quantity) and $\Delta \Delta E_{00}=\Delta E_{00}-\Delta E_{\text {ad }}$ denote their differences.

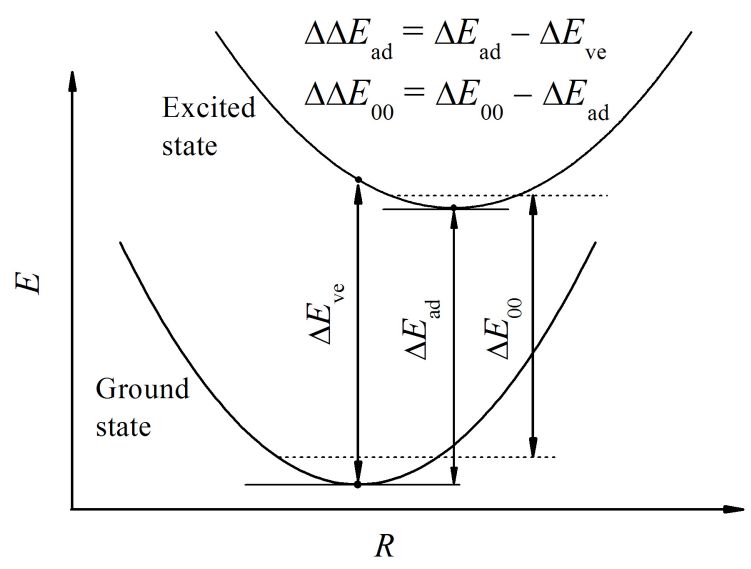

Figure 1. Quantities of interest in this work.

3.1. $\Delta E_{00}$ Energies with Different Methods. Although the primary aim of this work is not to assess the performance of excited-state methods in calculating experimentally available $\Delta E_{00}$ energies, but rather to investigate how computational estimates of the differences between $\Delta E_{\mathrm{ve}}, \Delta E_{\mathrm{ad}}$ and $\Delta E_{00}$ energies vary with the choice of method, it is nonetheless of interest to include in the presentation also the former analysis, especially since we are here using other methods or are examining other chemical systems than previous benchmarks on this topic. ${ }^{29,57}$ To this end, Table 2 shows the complete set (96 excited states, nine methods) of calculated $\Delta E_{00}$ energies. Furthermore, for each group of 
molecules as well as for data sets containing either all of the 96 excited states or all systems in groups I-III (inorganic molecules) and groups IV-IX (organic molecules), Table 2 also provides statistical analyses of the methods' mean absolute errors (MAEs), maximum absolute errors (MaxAEs) and mean signed errors (MSEs) relative to the experimental values. 


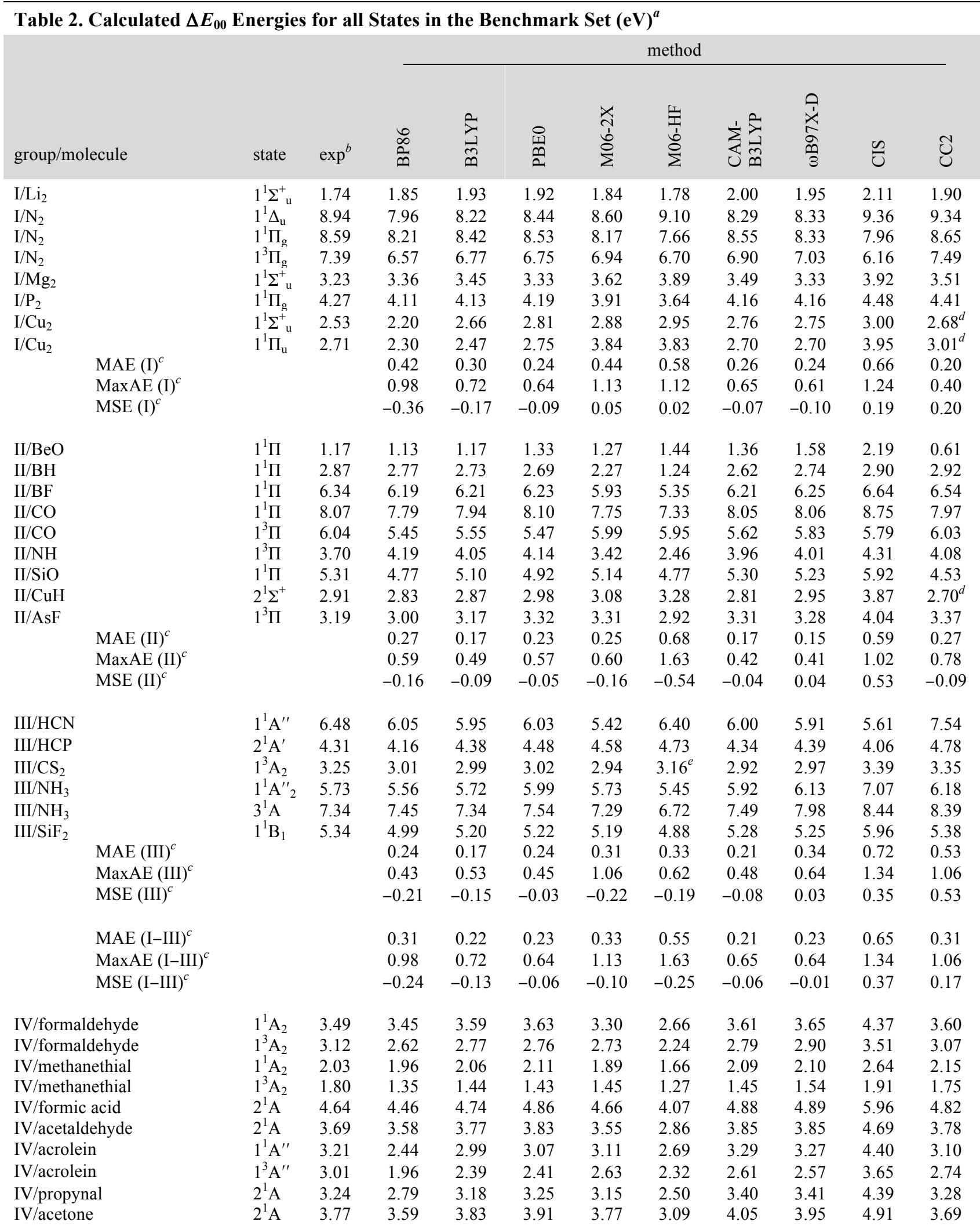


Table 2. Continued

\begin{tabular}{|c|c|c|c|c|c|c|c|c|c|c|c|}
\hline \multirow[b]{2}{*}{ group/molecule } & \multirow[b]{2}{*}{ state } & \multirow[b]{2}{*}{$\exp ^{b}$} & \multicolumn{9}{|c|}{ method } \\
\hline & & & $\begin{array}{l}\infty \\
\infty \\
\infty\end{array}$ & $\frac{\hat{\jmath}}{\tilde{\omega}}$ & $\begin{array}{l}\text { 피 } \\
\text { â }\end{array}$ & 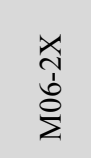 & 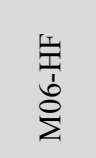 & 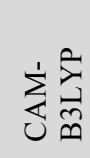 & $\begin{array}{l}\stackrel{0}{\dot{x}} \\
\stackrel{\hat{a}}{3} \\
\frac{\hat{3}}{3}\end{array}$ & $\stackrel{\Omega}{U}$ & U \\
\hline IV/thioacetone & $2^{1} \mathrm{~A}$ & 2.33 & 2.18 & 2.32 & 2.40 & 2.26 & 2.01 & 2.43 & 2.43 & 3.13 & 2.33 \\
\hline IV/thioacetone & $1^{3} \mathrm{~A}$ & 2.14 & 1.67 & 1.83 & 1.86 & 1.89 & 1.63 & 1.91 & 1.96 & 2.53 & 2.05 \\
\hline IV/glyoxal & $1^{1} \mathrm{~A}_{\mathrm{u}}$ & 2.72 & 1.96 & 2.38 & 2.41 & 2.37 & 1.87 & 2.59 & 2.56 & 3.53 & 2.74 \\
\hline IV/oxalyfluoride & $1^{1} \mathrm{~A}_{\mathrm{u}}$ & 4.02 & 3.21 & 3.66 & 3.78 & 3.82 & 3.28 & 3.96 & 3.95 & 5.15 & 4.02 \\
\hline $\operatorname{MAE}(\mathrm{IV})^{c}$ & & & 0.43 & 0.21 & 0.22 & 0.19 & 0.65 & 0.19 & 0.18 & 0.83 & 0.09 \\
\hline $\operatorname{MaxAE}(\mathrm{IV})^{c}$ & & & 1.05 & 0.62 & 0.60 & 0.39 & 0.88 & 0.40 & 0.44 & 1.32 & 0.27 \\
\hline $\operatorname{MSE}(\mathrm{IV})^{c}$ & & & -0.43 & -0.16 & -0.11 & -0.19 & -0.65 & -0.02 & -0.01 & 0.83 & -0.01 \\
\hline V/dichlorocarbene & $1^{1} \mathrm{~B}_{1}$ & 2.14 & 2.11 & 2.39 & 2.30 & $2.27^{f}$ & $2.27^{e}$ & 2.24 & 2.32 & 2.22 & 2.28 \\
\hline V/aminomethane & $2^{1} \mathrm{~A}^{\prime}$ & 5.18 & 4.93 & 5.21 & 5.52 & 5.40 & 5.04 & 5.41 & 5.61 & 6.59 & 5.74 \\
\hline V/aminomethane & $1^{1} \mathrm{~A}^{\prime \prime}$ & 6.22 & 6.14 & 6.25 & 6.57 & 6.56 & 6.18 & 6.52 & 6.75 & 8.00 & 7.26 \\
\hline V/aminoethane & $2^{1} \mathrm{~A}^{\prime}$ & 5.21 & 5.12 & 5.41 & 5.63 & 5.57 & 5.16 & 5.61 & 5.79 & 6.80 & 5.52 \\
\hline V/acetylene & $2^{1} \mathrm{~A}$ & 5.23 & 4.64 & 4.58 & 4.65 & 4.16 & 4.43 & 4.58 & 4.58 & 4.38 & 5.22 \\
\hline $\mathrm{V} /$ cyanoacetylene & $1^{1} \mathrm{~A}^{\prime \prime}$ & 4.77 & 4.39 & 4.69 & 4.46 & 5.12 & 5.33 & 4.81 & 4.81 & 5.05 & 5.21 \\
\hline $\mathrm{V} / \mathrm{hexatriene}$ & $1^{1} \mathrm{~B}_{\mathrm{u}}$ & 4.93 & 4.18 & 4.38 & 4.46 & 4.63 & 4.88 & 4.60 & 4.58 & 5.23 & 4.99 \\
\hline V/octatetraene & $1{ }^{1} \mathrm{~B}_{\mathrm{u}}$ & 4.41 & 3.55 & 3.76 & 3.83 & 4.02 & 4.31 & 4.01 & 4.01 & 4.66 & 4.33 \\
\hline $\operatorname{MAE}(\mathrm{V})^{c}$ & & & 0.38 & 0.31 & 0.40 & 0.40 & 0.23 & 0.31 & 0.40 & 0.82 & 0.33 \\
\hline $\operatorname{MaxAE}(\mathrm{V})^{c}$ & & & 0.86 & 0.65 & 0.58 & 1.07 & 0.80 & 0.65 & 0.65 & 1.78 & 1.04 \\
\hline $\operatorname{MSE}(\mathrm{V})^{c}$ & & & -0.38 & -0.18 & -0.08 & -0.05 & -0.06 & -0.04 & 0.05 & 0.61 & 0.31 \\
\hline VI/benzene & $1^{1} \mathrm{~B}_{2 \mathrm{u}}$ & 4.72 & 4.92 & 5.17 & 5.29 & 5.36 & 5.48 & 5.32 & 5.30 & 6.02 & 4.93 \\
\hline VI/toluene & $1^{1} \mathrm{~A}^{\prime \prime}$ & 4.65 & 4.79 & 5.05 & 5.16 & 5.26 & 5.41 & 5.22 & 5.20 & 5.94 & 4.85 \\
\hline VI/styrene & $2^{1} \mathrm{~A}$ & 4.31 & 4.12 & 4.51 & 4.60 & 4.78 & 5.00 & 4.73 & 4.73 & 5.19 & 4.57 \\
\hline VI/1,4-diethynylbenzene & $1^{1} \mathrm{~B}_{1 \mathrm{u}}$ & 4.25 & 3.87 & 4.21 & 4.31 & 4.57 & 4.76 & 4.47 & 4.47 & 4.94 & 4.45 \\
\hline $\mathrm{VI} /$ biphenyl & $1^{1} \mathrm{~B}_{1}$ & 4.37 & 4.21 & 4.32 & 4.43 & 4.62 & 4.89 & 4.57 & 4.59 & 5.01 & 4.54 \\
\hline VI/biphenylene & $1^{1} \mathrm{~B}_{1 \mathrm{u}}$ & 3.50 & 3.39 & 3.72 & 3.81 & 4.02 & 4.30 & 4.02 & 4.00 & 4.73 & 3.60 \\
\hline $\mathrm{VI} /$ trans-stilbene & $2^{1} \mathrm{~A}$ & 4.00 & 3.34 & 3.57 & 3.66 & 3.85 & 4.09 & 3.83 & 3.89 & 4.34 & 4.05 \\
\hline VI/naphthalene & $1^{1} \mathrm{~B}_{1 \mathrm{u}}$ & 3.97 & 3.71 & 4.00 & 4.10 & 4.35 & 4.66 & 4.42 & 4.29 & 4.85 & 4.15 \\
\hline VI/anthracene & $1^{1} \mathrm{~B}_{1 \mathrm{u}}$ & 3.43 & 2.64 & 2.92 & 3.01 & 3.28 & 3.65 & 3.26 & 3.27 & 3.91 & 3.38 \\
\hline VI/azulene & $1^{1} \mathrm{~B}_{2}$ & 1.77 & 1.85 & 1.98 & 2.02 & 2.03 & 2.05 & 2.07 & 2.06 & 2.69 & 1.87 \\
\hline VI/fluorine & $2{ }^{1} \mathrm{~A}_{1}$ & 4.19 & 4.01 & 4.25 & 4.37 & 4.56 & 4.79 & 4.51 & 4.52 & 4.91 & 4.37 \\
\hline VI/pyrene & $2^{1} \mathrm{~A}$ & 3.34 & 3.14 & 3.42 & 3.52 & 3.72 & 4.04 & 3.80 & 3.80 & 4.29 & 3.49 \\
\hline $\mathrm{VI} /$ terylene & $2^{1} \mathrm{~A}^{\prime}$ & 2.39 & 1.81 & 2.01 & 2.07 & 2.30 & 2.67 & 2.31 & 2.34 & 2.86 & 2.13 \\
\hline $\operatorname{MAE}(\mathrm{VI})^{c}$ & & & 0.30 & 0.24 & 0.28 & 0.35 & 0.53 & 0.34 & 0.32 & 0.83 & 0.16 \\
\hline $\operatorname{MaxAE}(\mathrm{VI})^{c}$ & & & 0.79 & 0.51 & 0.57 & 0.64 & 0.80 & 0.60 & 0.58 & 1.30 & 0.26 \\
\hline $\operatorname{MSE}(\mathrm{VI})^{c}$ & & & -0.24 & 0.02 & 0.11 & 0.29 & 0.53 & 0.28 & 0.27 & 0.83 & 0.11 \\
\hline VII/phenol & $2^{1} \mathrm{~A}$ & 4.51 & 4.45 & 4.79 & 4.90 & 5.05 & 5.22 & 4.99 & 4.97 & 5.81 & 4.66 \\
\hline VII/anisol & $2^{1} \mathrm{~A}$ & 4.51 & 4.37 & 4.78 & 4.89 & 5.05 & 5.23 & 4.99 & 4.97 & 5.81 & 4.64 \\
\hline VII/aniline & $2^{1} \mathrm{~A}$ & 4.22 & 3.96 & 4.38 & 4.49 & 4.68 & 4.86 & 4.64 & 4.63 & 5.55 & 4.39 \\
\hline VII/benzaldehyde & $1^{1} \mathrm{~A}^{\prime \prime}$ & 3.34 & 2.67 & 3.22 & 3.31 & 3.31 & 2.84 & 3.52 & 3.52 & 4.59 & 3.31 \\
\hline VII/benzaldehyde & $2^{1} \mathrm{~A}^{\prime}$ & 4.36 & 4.64 & 4.42 & 4.57 & 4.88 & 5.18 & 4.79 & 4.77 & 5.60 & 4.58 \\
\hline VII/benzaldehyde & $1^{3} \mathrm{~A}^{\prime \prime}$ & 3.12 & 2.21 & 2.64 & 2.67 & 2.82 & 2.46 & 2.89 & 2.91 & 3.84 & 2.94 \\
\hline VII/benzonitrile & $1^{1} \mathrm{~B}_{2}$ & 4.53 & 4.46 & 4.83 & 4.95 & 5.11 & 5.32 & 5.07 & 5.05 & 5.81 & 4.73 \\
\hline VII/4-aminobenzonitrile & $1^{1} \mathrm{~B}_{1}$ & 4.15 & 3.85 & 4.30 & 4.41 & 4.60 & 4.81 & 4.58 & 4.55 & 5.45 & 4.30 \\
\hline VII/DMA-benzonitrile & $1^{1} \mathrm{~B}$ & 4.02 & 3.54 & 4.09 & 4.21 & 4.47 & 4.71 & 4.46 & 4.47 & 5.39 & 4.06 \\
\hline VII/hydroquinone & $1^{1} \mathrm{~B}_{\mathrm{u}}$ & 4.15 & 3.94 & 4.32 & 4.42 & 4.60 & 4.82 & 4.52 & 4.51 & 5.45 & 4.24 \\
\hline VII/1,4-phenylenediamine & $2^{1} \mathrm{~A}$ & 3.70 & 3.41 & 3.81 & 3.90 & 4.09 & 4.30 & 4.05 & 4.04 & 5.05 & 3.92 \\
\hline VII/DMA-aniline & $2^{1} \mathrm{~A}$ & 3.64 & 3.26 & 3.71 & 3.81 & 4.04 & 4.27 & 3.99 & 3.98 & 5.02 & 3.76 \\
\hline VII/methyl 4-coumarate & $2^{1} \mathrm{~A}$ & 4.08 & 3.58 & 3.90 & 4.00 & 4.19 & 4.41 & 4.17 & 4.19 & 4.83 & 4.20 \\
\hline VII/DMA-cyanostilbene & $2^{1} \mathrm{~A}$ & 3.36 & 2.50 & 2.97 & 3.07 & 3.32 & 3.61 & 3.35 & 3.40 & 3.97 & 3.25 \\
\hline $\operatorname{MAE}(\mathrm{VII})^{c}$ & & & 0.39 & 0.20 & 0.26 & 0.38 & 0.62 & 0.34 & 0.34 & 1.18 & 0.14 \\
\hline
\end{tabular}




\begin{tabular}{|c|c|c|c|c|c|c|c|c|c|c|c|}
\hline \multirow[b]{2}{*}{ group/molecule } & \multirow[b]{2}{*}{ state } & \multirow[b]{2}{*}{$\exp ^{b}$} & \multicolumn{9}{|c|}{ method } \\
\hline & & & $\begin{array}{l}\infty \\
\infty \\
\infty\end{array}$ & $\frac{\hat{\jmath}}{\tilde{\omega}}$ & $\begin{array}{l}\text { 피 } \\
\text { â }\end{array}$ & 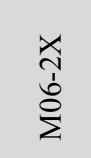 & 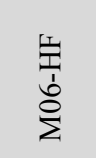 & 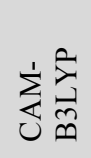 & $\begin{array}{l}\hat{1} \\
\dot{x} \\
\hat{a} \\
\hat{3}\end{array}$ & $\tilde{U}$ & లુ \\
\hline $\operatorname{MaxAE}(\mathrm{VII})^{c}$ & & & 0.91 & 0.48 & 0.45 & 0.58 & 0.82 & 0.54 & 0.52 & 1.38 & 0.22 \\
\hline $\operatorname{MSE}(\mathrm{VII})^{c}$ & & & -0.35 & 0.03 & 0.14 & 0.32 & 0.45 & 0.31 & 0.31 & 1.18 & 0.09 \\
\hline VIII/pyridine & $2^{1} \mathrm{~A}$ & 4.31 & 3.76 & 4.18 & 4.25 & 4.22 & 3.93 & 4.42 & 4.39 & 5.44 & 4.37 \\
\hline VIII/pyrimidine & $2^{1} \mathrm{~A}$ & 3.85 & 3.29 & 3.69 & 3.77 & 3.80 & 3.74 & 3.96 & 3.93 & 5.26 & 3.89 \\
\hline VIII/pyrimidine & $2^{1} \mathrm{~A}_{2}$ & 5.00 & 4.50 & 5.07 & 5.17 & 5.03 & 5.57 & 5.38 & 5.37 & 5.50 & 5.13 \\
\hline VIII/2-chloropyrimidine & $2^{1} \mathrm{~A}^{\prime}$ & 3.98 & 3.32 & 3.76 & 3.86 & 3.89 & 3.84 & 4.03 & 4.01 & 5.35 & 3.93 \\
\hline VIII/pyridone lactim & $2^{1} \mathrm{~A}$ & 4.48 & 4.32 & 4.73 & 4.84 & 4.98 & 4.53 & 4.92 & 4.91 & 5.80 & 4.57 \\
\hline VIII/pyridone lactam & $2^{1} \mathrm{~A}$ & 3.70 & 2.74 & 3.80 & 3.84 & 4.14 & 4.28 & 4.09 & 4.08 & 4.99 & 3.70 \\
\hline VIII/tetrazine & $2^{1} \mathrm{~A}$ & 2.25 & 1.63 & 1.98 & 1.99 & 1.97 & 1.85 & 2.17 & 2.13 & 3.21 & 2.26 \\
\hline VIII/indole & $2^{1} \mathrm{~A}^{\prime}$ & 4.37 & 3.94 & 4.37 & 4.50 & 4.75 & 5.04 & 4.72 & 4.71 & 5.40 & 4.54 \\
\hline VIII/7-azaindole & $2^{1} \mathrm{~A}$ & 4.29 & 3.68 & 4.13 & 4.27 & 4.57 & 4.35 & 4.54 & 4.52 & 5.34 & 4.44 \\
\hline VIII/cinnoline & $1^{1} \mathrm{~A}^{\prime \prime}$ & 2.82 & 1.84 & 2.42 & 2.48 & 2.60 & 2.40 & 2.76 & 2.75 & 3.81 & 2.81 \\
\hline VIII/quinoline & $2^{1} \mathrm{~A}$ & 3.99 & 2.92 & 3.52 & 3.61 & 3.73 & 3.62 & 3.88 & 3.84 & 4.98 & 3.71 \\
\hline VIII/quinoline & $1^{3} \mathrm{~A}^{\prime}$ & 2.79 & 2.34 & 2.25 & 2.09 & 2.70 & 3.04 & $2.08^{g}$ & 2.13 & 2.30 & 2.82 \\
\hline VIII/quinoxaline & $2^{1} \mathrm{~A}$ & 3.36 & 2.53 & 3.05 & 3.11 & 3.21 & 3.17 & 3.38 & 3.34 & 4.54 & 3.34 \\
\hline VIII/quinoxaline & $2^{1} \mathrm{~A}_{1}$ & 3.97 & 3.91 & 4.18 & 4.28 & 4.38 & 4.62 & 4.36 & 4.34 & 5.13 & 4.11 \\
\hline VIII/quinoxaline & $1^{3} \mathrm{~A}$ & 2.68 & 2.14 & 2.19 & 2.05 & 2.80 & 2.80 & $2.03^{g}$ & 2.07 & 2.27 & 2.79 \\
\hline VIII/ $\beta$-dinaphthyleneoxide & $2^{1} \mathrm{~A}$ & 3.63 & 2.99 & 3.26 & 3.37 & 3.65 & 4.02 & 3.64 & 3.65 & 4.24 & 3.63 \\
\hline $\mathrm{VIII} /$ syn-coumarin 153 & $2^{1} \mathrm{~A}$ & 3.21 & 2.41 & 2.99 & 3.14 & 3.51 & 3.77 & 3.52 & 3.55 & 4.46 & 3.04 \\
\hline VIII/porphyrin & $1^{1} \mathrm{~B}_{1 \mathrm{u}}$ & 2.02 & 2.03 & 2.18 & 2.24 & 2.25 & 1.99 & 2.18 & 2.10 & 2.59 & 2.22 \\
\hline VIII/merocyanine dye & $2^{1} \mathrm{~A}$ & 2.58 & 1.80 & 2.52 & 2.74 & 2.98 & 2.89 & 3.09 & 3.09 & 3.97 & 2.82 \\
\hline MAE $(\mathrm{VIII})^{c}$ & & & 0.59 & 0.24 & 0.25 & 0.23 & 0.33 & 0.27 & 0.26 & 1.01 & 0.10 \\
\hline $\operatorname{MaxAE}(\mathrm{VIII})^{c}$ & & & 1.07 & 0.54 & 0.70 & 0.50 & 0.67 & 0.71 & 0.66 & 1.41 & 0.28 \\
\hline $\operatorname{MSE}(\mathrm{VIII})^{c}$ & & & -0.59 & -0.16 & -0.09 & 0.10 & 0.11 & 0.10 & 0.09 & 0.91 & 0.04 \\
\hline IX/2-cyclopenten-1-one & $2^{1} \mathrm{~A}$ & 3.37 & 2.63 & 3.22 & 3.33 & 3.42 & 3.00 & 3.59 & 3.57 & 4.81 & 3.18 \\
\hline IX/1,4-benzoquinone & $1^{1} \mathrm{~B}_{1 \mathrm{~g}}$ & 2.49 & 1.65 & 2.22 & 2.28 & 2.48 & 2.19 & 2.65 & 2.62 & 3.95 & $-{ }^{h}$ \\
\hline IX/1,4-benzoquinone & $1^{1} \mathrm{~B}_{3 \mathrm{~g}}$ & 4.07 & 2.85 & 3.25 & 3.37 & 3.80 & 4.39 & 3.80 & 3.59 & 4.93 & $-^{h}$ \\
\hline IX/DBH & $2^{1} \mathrm{~A}$ & 3.66 & 3.37 & 3.46 & 3.53 & 3.26 & 2.77 & 3.51 & 3.53 & 4.01 & 3.81 \\
\hline IX/1,6-epoxy-10-annulene & $1^{1} \mathrm{~B}_{1}$ & 2.98 & 3.12 & 3.30 & 3.40 & 3.42 & 3.49 & 3.37 & 3.37 & 3.93 & 3.16 \\
\hline $\operatorname{MAE}(\mathrm{IX})^{c}$ & & & 0.65 & 0.35 & 0.30 & 0.23 & 0.48 & 0.24 & 0.27 & 1.01 & 0.17 \\
\hline $\operatorname{MaxAE}(\mathrm{IX})^{c}$ & & & 1.22 & 0.82 & 0.70 & 0.44 & 0.89 & 0.39 & 0.48 & 1.46 & 0.19 \\
\hline $\operatorname{MSE}(\mathrm{IX})^{c}$ & & & -0.59 & -0.22 & -0.13 & -0.04 & -0.15 & 0.07 & 0.02 & 1.01 & 0.05 \\
\hline MAE $(\mathrm{IV}-\mathrm{IX})^{c}$ & & & 0.45 & 0.24 & 0.27 & 0.29 & 0.48 & 0.28 & 0.28 & 0.95 & 0.15 \\
\hline $\operatorname{MaxAE}(\mathrm{IV}-\mathrm{IX})^{c}$ & & & 1.22 & 0.82 & 0.70 & 1.07 & 0.89 & 0.71 & 0.66 & 1.78 & 1.04 \\
\hline $\operatorname{MSE}(\mathrm{IV}-\mathrm{IX})^{c}$ & & & -0.43 & -0.10 & -0.02 & 0.10 & 0.07 & 0.13 & 0.13 & 0.90 & 0.09 \\
\hline $\operatorname{MAE}(\mathrm{I}-\mathrm{IX})^{c}$ & & & 0.42 & 0.24 & 0.26 & 0.30 & 0.50 & 0.27 & 0.27 & 0.88 & 0.19 \\
\hline $\operatorname{MaxAE}(\mathrm{I}-\mathrm{IX})^{c}$ & & & 1.22 & 0.82 & 0.70 & 1.13 & 1.63 & 0.71 & 0.66 & 1.78 & 1.06 \\
\hline $\operatorname{MSE}(\mathrm{I}-\mathrm{IX})^{c}$ & & & -0.38 & -0.11 & -0.03 & 0.05 & -0.01 & 0.09 & 0.10 & 0.78 & 0.11 \\
\hline
\end{tabular}

${ }^{a}$ All calculations required to obtain $\Delta E_{00}$ energies performed using the cc-pVDZ basis set. ${ }^{b}$ Experimental values compiled in reference 29, see also references therein. ${ }^{c}$ Mean absolute error (MAE), maximum absolute error (MaxAE), and mean signed error (MSE) relative to the experimental values for all states in the indicated group(s) of molecules. ${ }^{d}$ Because of the unavailability of an auxiliary cc-pVDZ basis set for $\mathrm{Cu}$ in TURBOMOLE 6.3, this value was derived from CCSD/cc-pVDZ and EOM-CCSD/cc-pVDZ calculations with GAUSSIAN 09. ${ }^{e}$ Since the M06-HF ground-state geometry optimization failed to converge, this value was obtained from M06 calculations. ${ }^{f}$ Since the M06-2X ground-state geometry optimization failed to converge, this value was obtained from M06 calculations. ${ }^{g}$ Since the CAM-B3LYP ground-state geometry optimization failed to converge, this value was obtained from HSE06 calculations. ${ }^{h} \mathrm{CC} 2$ ground-state geometry optimization failed to converge. ${ }^{i}$ Value excluding the $\mathrm{CC} 2 \Delta E_{00}$ energies for the $1^{1} \mathrm{~B}_{1 \mathrm{~g}}$ and $1{ }^{1} \mathrm{~B}_{3 \mathrm{~g}}$ states of 1 ,4-benzoquinone. 
Starting with the small inorganic molecules of groups I-III, the best performance is achieved by the B3LYP and PBE0 global hybrid GGAs and the CAM-B3LYP and $\omega$ B97X-D long-range-corrected hybrid GGAs (MAEs of $0.21-0.23 \mathrm{eV}$ ). Unsurprisingly, at the other end of the spectrum we find CIS (MAE of $0.65 \mathrm{eV}$ ), whose tendency to overestimate excitation energies is well known ${ }^{13,34}$ and is reflected in a positive MSE of $0.37 \mathrm{eV}$. In the intermediate range, the BP86 pure GGA, the M06-2X and M06-HF global hybrid meta-GGAs, and the ab initio CC2 method show MAEs of $0.31-0.55 \mathrm{eV}$. Notably, the methods with the smallest MAEs are also the most robust ones as measured by their MaxAE values. Specifically, the MaxAEs fall within the $0.64-0.72 \mathrm{eV}$ range for B3LYP, PBE0, CAM-B3LYP and $\omega$ B97X-D; amount to $0.98-1.13 \mathrm{eV}$ for BP86, M06-2X and $\mathrm{CC} 2$; and reach a substantial $1.34-1.63 \mathrm{eV}$ for CIS and M06-HF.

For the organic molecules of groups IV-IX, in turn, CC2 affords a MAE of a mere $0.15 \mathrm{eV}$ that none of the DFT methods can rival, albeit that five of them - B3LYP, PBE0, M06-2X, CAM-B3LYP and $\omega$ B97X-D - perform quite well (MAEs of 0.24-0.29 eV). The largest MAEs are shown by the BP86 $(0.45 \mathrm{eV})$, M06-HF $(0.48 \mathrm{eV})$ and CIS $(0.95 \mathrm{eV})$ methods. The marked improvement of $\mathrm{CC} 2$ compared to how this method fared for the inorganic molecules is likely to reflect the relative unimportance of multireference correlation effects for most of the organic excited states studied, whereas for the inorganic systems, at least a few states (e.g., the $1^{1} \Pi$ state of $\mathrm{SiO}^{86}$ ) have some double-excitation character or are populated from a multi-reference ground state (as identifiable by various diagnostics ${ }^{87-89}$ ).

Further attesting to the accuracy of $\mathrm{CC} 2$ for organic molecules is the observation that this method exhibits both the smallest MAEs and the smallest MaxAEs for all groups 
of organic molecules except group V (hydrocarbons). Specifically, for groups IV and VIIX, CC2 shows MAEs of $0.09-0.17 \mathrm{eV}$ and MaxAEs of $0.19-0.28 \mathrm{eV}$ only. For group V, the $\mathrm{CC} 2$ statistics are notably worse (MAE of $0.33 \mathrm{eV}$ and MaxAE of $1.04 \mathrm{eV}$ ), but primarily because of a hefty $1.04 \mathrm{eV}$ overestimation of a single $\Delta E_{00}$ energy (the $1^{1} \mathrm{~A}^{\prime \prime}$ state of aminomethane). In part, this error may be due to some admixture of Rydberg character into the $1^{1} \mathrm{~A}^{\prime \prime}$ state, ${ }^{29}$ although the present calculations (using population analysis) give no indication that this effect is pronounced.

It is also of interest to evaluate whether the computational methods overall tend to over- or underestimate the $\Delta E_{00}$ energies of the different groups of molecules by examining the corresponding MSE values. Compared with $\Delta E_{\mathrm{ve}}$-based benchmarks that neglect geometric relaxation effects in the excited state and therefore are inclined to exaggerate (understate) the tendency of a particular method to yield too high (low) excitation energies compared with experimental absorption maxima, the full inclusion of geometric relaxation effects may render the present analysis oppositely inclined, despite that the comparison is here with experimental $\Delta E_{00}$ energies.

Starting with the inorganic molecules of groups I-III, all density functionals show negative MSEs and thus tend to underestimate these $\Delta E_{00}$ energies. In contrast, the ab initio CIS and CC2 methods show positive MSEs. Continuing with the organic molecules of groups IV-IX, BP86 displays a distinctly negative MSE of $-0.43 \mathrm{eV}$. Among the hybrid functionals, those that include a small fraction of exact exchange display negative or slightly negative MSEs of -0.10 (B3LYP) and $-0.02 \mathrm{eV}$ (PBE0), whereas those that include a large fraction show positive MSEs of $0.07-0.13 \mathrm{eV}$ (M06-2X, M06-HF, CAMB3LYP and $\omega \mathrm{B} 97 \mathrm{X}-\mathrm{D})$. These trends are consistent with the observation in a previous 
TD-DFT benchmark concerned entirely with $\Delta E_{\mathrm{ve}}$ energies that (albeit with some notable exceptions $^{90}$ ) pure functionals like BP86 typically red-shift, and the inclusion of successively larger fractions of exact exchange in hybrid functionals typically blue-shift, the excitation energies of organic molecules. ${ }^{12}$ CIS and $\mathrm{CC} 2$, in turn, show positive MSEs for the organic systems.

As for the statistical analyses in Table 2 involving all of the 96 excited states included in the benchmark, three key observations can be made. First, the overall bestperforming method is CC2 (MAE of $0.19 \mathrm{eV})$, followed by B3LYP $(0.24 \mathrm{eV})$, PBE0 (0.26 eV), CAM-B3LYP (0.27 eV), $\omega B$ B7X-D (0.27 eV), M06-2X (0.30 eV), BP86 (0.42 eV), M06-HF $(0.50 \mathrm{eV})$ and, lastly, CIS $(0.88 \mathrm{eV})$. That B3LYP and PBE0 are suitable density functionals for calculating $\Delta E_{00}$ energies is consistent with the results of Furche and co-workers, ${ }^{29}$ who employed a somewhat different computational strategy than us (see comments in the Introduction). The benchmark study by Jacquemin and coworkers ${ }^{57}$ who exclusively looked at large conjugated dyes in solution and thus were faced with the additional challenge to reliably describe solvent effects, altogether favored M06-2X and CAM-B3LYP over B3LYP and PBE0, albeit without finding major differences in the corresponding MAEs.

Second, while B3LYP for the 96 excited states by a narrow $0.02 \mathrm{eV}$ margin exhibits the smallest MAE among the different functionals, the B3LYP MaxAE of 0.82 $\mathrm{eV}$ is larger than those of $\omega \mathrm{B} 97 \mathrm{X}-\mathrm{D}(0.66 \mathrm{eV})$, PBE0 $(0.70 \mathrm{eV})$ and CAM-B3LYP $(0.71$ eV). Thus, the latter methods appear slightly more robust than B3LYP.

Third, the least accurate functional for the current benchmark set is M06-HF (MAE of $0.50 \mathrm{eV}$ ), despite that this method has a wider range of applicability to different 
types of excited states (including Rydberg and charge-transfer states) than most other functionals. ${ }^{10,69}$ Since the majority of the 96 states have valence character, this finding corroborates the observation made in a recent $\Delta E_{\mathrm{ve}}$-based benchmark that M06-HF is not very well suited for valence excitations. ${ }^{15}$ In this regard, it is of course possible that the M06-HF results are negatively affected by the procedure that all calculations required to obtain $\Delta E_{00}$ energies with a given method are carried out using that particular method, including not only the singlepoint calculations but also the geometry optimizations and frequency calculations. However, we believe that this procedure is the one that most fully reflects a method's appropriateness for calculating $\Delta E_{00}$ energies.

Table 3, finally, lists correlation coefficients between the calculated and experimental $\Delta E_{00}$ energies for all states in Table 2. For two data sets $X$ and $Y$ with elements $\{x\}$ and $\{y\}$, the correlation coefficient $\rho(X, Y)$ was computed as

$$
\rho(X, Y)=\frac{\sum(x-\bar{x})(y-\bar{y})}{\sqrt{\sum(x-\bar{x})^{2} \sum(y-\bar{y})^{2}}},
$$

where $\bar{x}$ and $\bar{y}$ are the arithmetic means of the data sets and the summations run over all elements. As can be seen, the ranking that this measure provides of how well the different methods perform matches the aforementioned ranking based on MAEs. Indeed, the largest $\rho$ value is achieved by CC2 (0.988), followed by B3LYP (0.981), PBE0 (0.977), CAM-B3LYP (0.977), $\omega B 97 X-D ~(0.977)$, BP86 (0.972), M06-2X (0.967), M06-HF (0.922) and, lastly, CIS (0.918). Through the $\rho$ values in Table 3, it is further possible to compare how similar the $\Delta E_{00}$ energies obtained with any two methods are to each other. 
For example, among the DFT methods, we observe particularly strong correlations between the B3LYP, PBE0, CAM-B3LYP and $\omega$ B97X-D results, with a $\rho$ value of $\geq 0.994$ for any comparison among these data sets. Furthermore, we also note that the M06-HF results are the ones that correlate the least with those of other DFT methods.

\begin{tabular}{|c|c|c|c|c|c|c|c|c|c|c|}
\hline \multirow[b]{2}{*}{ method } & \multicolumn{10}{|c|}{ method } \\
\hline & : & $\begin{array}{l}\infty \\
\infty \\
\stackrel{\infty}{\infty}\end{array}$ & $\frac{\partial}{\vec{\lambda}}$ & $\begin{array}{l}\text { 이 } \\
\text { ma } \\
\text { a }\end{array}$ & \begin{tabular}{l}
$x$ \\
\multirow{1}{*}{} \\
$\delta$ \\
$\Sigma$
\end{tabular} & 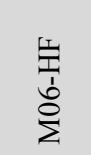 & 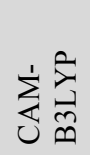 & 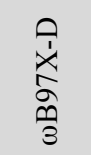 & 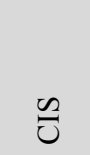 & ঠ \\
\hline $\exp ^{a}$ & 1 & 0.972 & 0.981 & 0.977 & 0.967 & 0.922 & 0.977 & 0.977 & 0.918 & 0.988 \\
\hline BP86 & 0.972 & 1 & 0.990 & 0.987 & 0.963 & 0.910 & 0.975 & 0.977 & 0.903 & 0.978 \\
\hline B3LYP & 0.981 & 0.990 & 1 & 0.998 & 0.982 & 0.931 & 0.994 & 0.994 & 0.938 & 0.980 \\
\hline PBE0 & 0.977 & 0.987 & 0.998 & 1 & 0.983 & 0.932 & 0.995 & 0.996 & 0.948 & 0.979 \\
\hline M06-2X & 0.967 & 0.963 & 0.982 & 0.983 & 1 & 0.970 & 0.987 & 0.986 & 0.948 & 0.965 \\
\hline M06-HF & 0.922 & 0.910 & 0.931 & 0.932 & 0.970 & 1 & 0.942 & 0.937 & 0.891 & 0.923 \\
\hline CAM-B3LYP & 0.977 & 0.975 & 0.994 & 0.995 & 0.987 & 0.942 & 1 & 0.998 & 0.959 & 0.973 \\
\hline$\omega B 97 X-D$ & 0.977 & 0.977 & 0.994 & 0.996 & 0.986 & 0.937 & 0.998 & 1 & 0.959 & 0.975 \\
\hline CIS & 0.918 & 0.903 & 0.938 & 0.948 & 0.948 & 0.891 & 0.959 & 0.959 & 1 & 0.913 \\
\hline $\mathrm{CC} 2$ & 0.988 & 0.978 & 0.980 & 0.979 & 0.965 & 0.923 & 0.973 & 0.975 & 0.913 & 1 \\
\hline
\end{tabular}

\subsection{Variation Between Methods in their Estimates of $\Delta \Delta E_{\text {ad }}$ and $\Delta \Delta E_{00}$ Energy}

Differences. Having assessed how well different excited-state methods perform in calculating $\Delta E_{00}$ energies, we now turn to analyzing the variation between methods in their estimates of $\Delta \Delta E_{\text {ad }}=\Delta E_{\text {ad }}-\Delta E_{\mathrm{ve}}$ and $\Delta \Delta E_{00}=\Delta E_{00}-\Delta E_{\text {ad }}$ energy differences. To put the analysis in proper perspective, a comparison is made with how much the excitation energies themselves vary with the method. Specifically, we have used the variation in $\Delta E_{\mathrm{ve}}$ energies as reference, but could equally well have used the variation in $\Delta E_{\text {ad }}$ or $\Delta E_{00}$ energies (this choice is of no consequence for the conclusions drawn). In our opinion, it is natural to choose $\Delta E_{\mathrm{ve}}$ energies as reference as they constitute the 
starting point from which the $\Delta \Delta E_{\text {ad }}$ excited-state relaxation energies are calculated. Since the particular features of any given method to some extent will affect all three kinds of excitation energies in a similar way, the $\Delta \Delta E_{\text {ad }}$ and $\Delta \Delta E_{00}$ energy differences are of course expected to vary less between methods than the $\Delta E_{\mathrm{ve}}$ energies; however, an attempt to quantify this relationship is largely missing in the existing literature. Thus, the present analysis will help filling an important gap.

The results of the analysis, pertaining to calculations with the BP86, B3LYP, PBE0, M06-2X, M06-HF, CAM-B3LYP, wB97X-D, CIS and CC2 methods, are summarized in Figures $2 \mathrm{a}-2 \mathrm{c}$ and $3 \mathrm{a}-3 \mathrm{c}$, and in Table 4. Figures $2 \mathrm{a}-2 \mathrm{c}$ compare standard deviations (SDs), obtained as

$\sigma(X)=\sqrt{\frac{\sum(x-\bar{x})^{2}}{n}}$,

for calculated $\Delta \Delta E_{\text {ad }}$ and $\Delta \Delta E_{00}$ energy differences with the corresponding data for $\Delta E_{\mathrm{ve}}$ energies. Figures $3 \mathrm{a}-3 \mathrm{c}$, in turn, present an analogous comparison of maximum absolute deviations (MaxADs). In both sets of figures, the SD and MaxAD descriptors are shown for all individual excited states included in the different groups of the benchmark set, whereas the $\Delta E_{\mathrm{ve}}, \Delta \Delta E_{\mathrm{ad}}$ and $\Delta \Delta E_{00}$ data underlying the analysis are given in Tables S1S9 of the Supporting Information. Table 4, finally, lists the average values of the SDs (denoted ASD) and the maximum values of the MaxADs (denoted GMaxAD) within each group, as well as within groups I-III (inorganic molecules), groups IV-IX (organic molecules), and groups I-IX (the full benchmark set) merged together. 

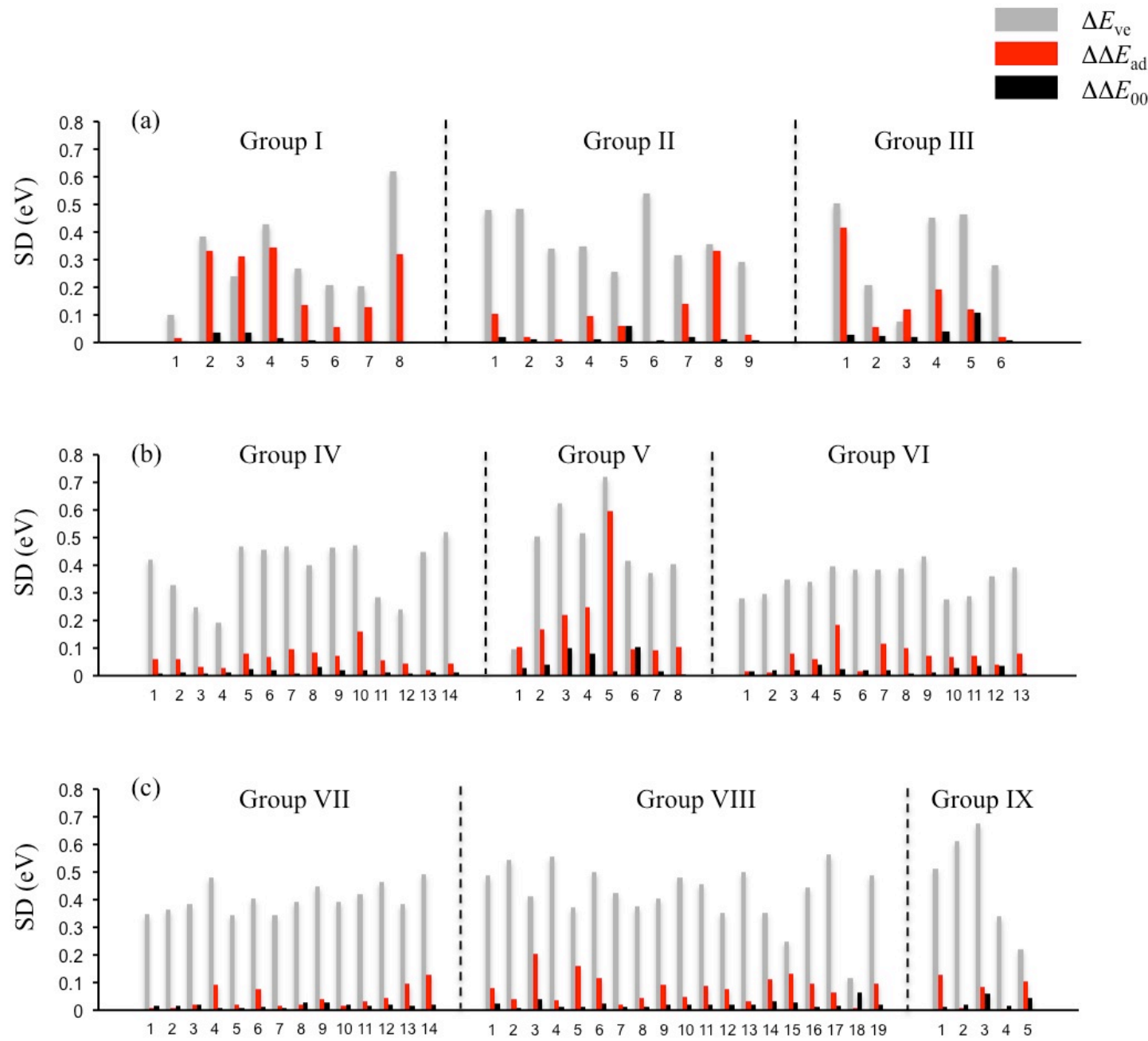

Figure 2. Standard deviations for calculated $\Delta E_{\mathrm{ve}}$ energies and $\Delta \Delta E_{\mathrm{ad}}$ and $\Delta \Delta E_{00}$ energy differences of individual states in groups I-III (a), IV-VI (b), and VII-IX (c) of the benchmark set using the BP86, B3LYP, PBE0, M06-2X, M06-HF, CAM-B3LYP, $\omega \mathrm{B} 97 \mathrm{X}-\mathrm{D}, \mathrm{CIS}$ and CC2 methods. For each group, states are labeled following Table 1. 

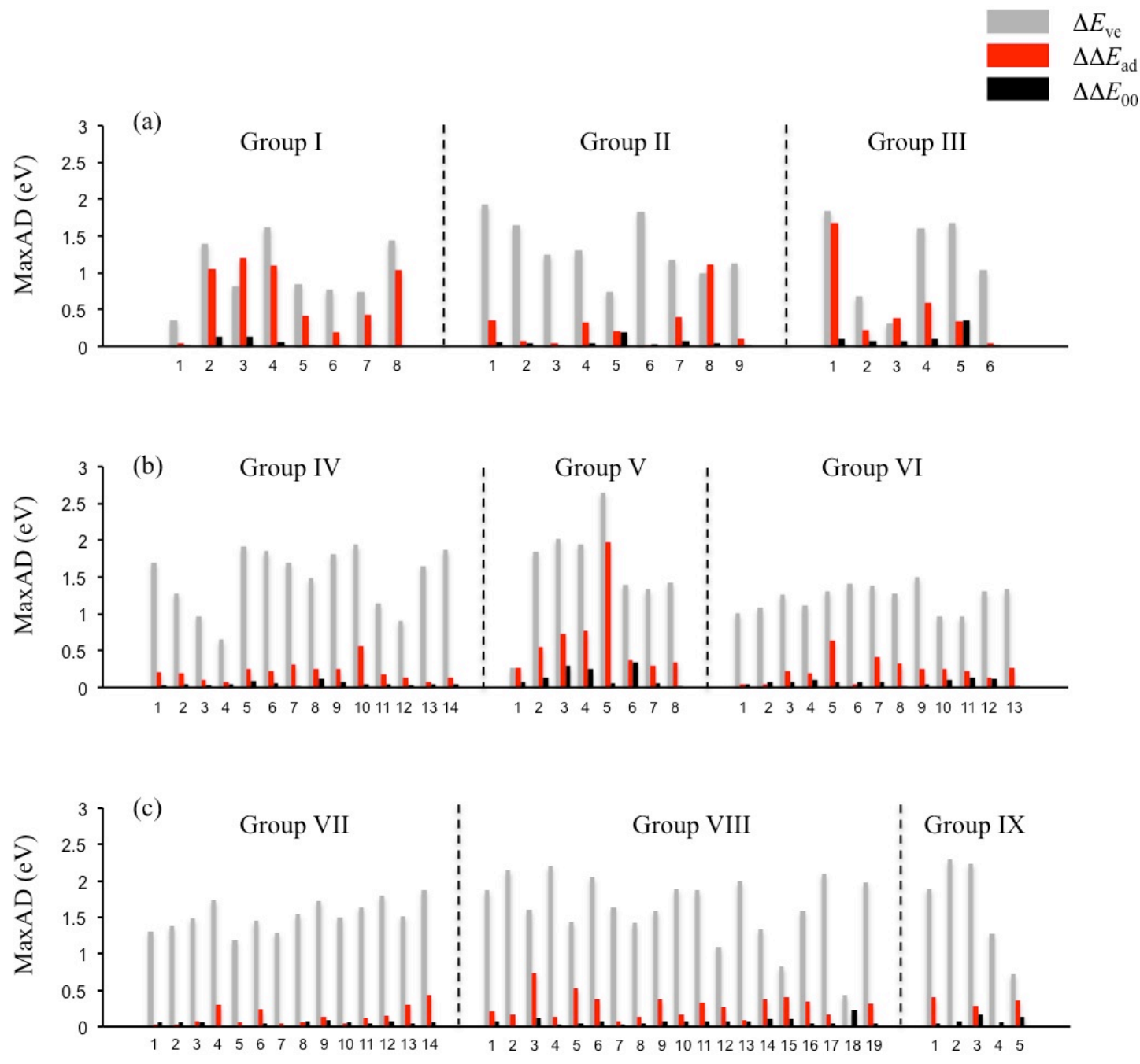

Figure 3. Maximum absolute deviations for calculated $\Delta E_{\mathrm{ve}}$ energies and $\Delta \Delta E_{\mathrm{ad}}$ and $\Delta \Delta E_{00}$ energy differences of individual states in groups I-III (a), IV-VI (b), and VII-IX (c) of the benchmark set using the BP86, B3LYP, PBE0, M06-2X, M06-HF, CAMB3LYP, $\omega$ B97X-D, CIS and CC2 methods. For each group, states are labeled following Table 1. 
Table 4. ASD and GMaxAD Values for Calculated $\Delta E_{\mathrm{ve}}$ Energies and $\Delta \Delta E_{\text {ad }}$ and $\Delta \Delta E_{00}$ Energy Differences of all States in Different Groups of the Benchmark Set (eV)

\begin{tabular}{|c|c|c|c|c|c|c|}
\hline \multirow[b]{2}{*}{ group(s) } & \multicolumn{2}{|c|}{$\Delta E_{\mathrm{ve}}$} & \multicolumn{2}{|c|}{$\Delta \Delta E_{\text {ad }}$} & \multicolumn{2}{|c|}{$\Delta \Delta E_{00}$} \\
\hline & $\mathrm{ASD}^{a}$ & $\overline{G M a x A D}^{a}$ & $\mathrm{ASD}^{a}$ & $\overline{\operatorname{GMaxAD}}^{a}$ & $\mathrm{ASD}^{a}$ & $\mathrm{GMaxAD}^{a}$ \\
\hline I & 0.31 & 1.62 & 0.21 & 1.20 & 0.01 & 0.13 \\
\hline II & 0.38 & 1.93 & 0.09 & 1.11 & 0.02 & 0.20 \\
\hline III & 0.33 & 1.84 & 0.15 & 1.68 & 0.04 & 0.36 \\
\hline IV & 0.39 & 1.94 & 0.06 & 0.56 & 0.02 & 0.12 \\
\hline $\mathrm{V}$ & 0.46 & 2.65 & 0.20 & 1.97 & 0.05 & 0.34 \\
\hline VI & 0.35 & 1.50 & 0.07 & 0.64 & 0.02 & 0.13 \\
\hline VII & 0.40 & 1.87 & 0.04 & 0.44 & 0.02 & 0.09 \\
\hline VIII & 0.42 & 2.20 & 0.08 & 0.74 & 0.02 & 0.23 \\
\hline IX & 0.47 & 2.29 & 0.07 & 0.40 & 0.03 & 0.17 \\
\hline I-III & 0.34 & 1.93 & 0.15 & 1.68 & 0.02 & 0.36 \\
\hline IV-IX & 0.41 & 2.65 & 0.08 & 1.97 & 0.02 & 0.34 \\
\hline I-IX & 0.39 & 2.65 & 0.10 & 1.97 & 0.02 & 0.36 \\
\hline
\end{tabular}

From Table 4, it can be seen that while the ASD for the full benchmark set is 0.39 $\mathrm{eV}$ for the calculation of $\Delta E_{\mathrm{ve}}$ energies, the estimation of $\Delta \Delta E_{\mathrm{ad}}$ and (particularly) $\Delta \Delta E_{00}$ energy differences is much less sensitive to the choice of computational method, as evidenced by ASDs of a mere 0.10 and $0.02 \mathrm{eV}$, respectively. This trend is manifested for both the organic systems of groups IV-IX (somewhat more pronouncedly) and the inorganic systems of groups I-III (somewhat less pronouncedly), with ASDs of 0.41 $\left(\Delta E_{\mathrm{ve}}\right), 0.08\left(\Delta \Delta E_{\text {ad }}\right)$ and $0.02 \mathrm{eV}\left(\Delta \Delta E_{00}\right)$ for the former molecules and of $0.34,0.15$ and $0.02 \mathrm{eV}$ for the latter. This demonstrates that photochemical problems requiring accurate estimates of $\Delta \Delta E_{\text {ad }}$ and $\Delta \Delta E_{00}$ energy differences can be approached using methods that are less elaborate than those oftentimes needed for accurate calculation of excitation energies. At the same time, while the overall GMaxAD value of $0.36 \mathrm{eV}$ shows that it is possible to calculate $\Delta \Delta E_{00}$ for all excited states in the benchmark set using the cheapest 
and least accurate method (here CIS) without the results being very different from the results obtained with the most expensive and reliable method (here CC2), it is similarly clear that the calculation of $\Delta \Delta E_{\mathrm{ad}}$ relaxation energies can be sensitive to the choice of method. For example, for each of groups I-III, the corresponding GMaxAD is $1.11 \mathrm{eV}$ or larger.

Out of the above results, it can be argued that it is not surprising (but nonetheless worth establishing) that $\Delta \Delta E_{00}$ varies so little between methods, as this quantity is simply the difference in ZPVE corrections between the excited state and the ground state. Furthermore, while there is an obvious positive implication of this result for the calculation and comparison of ground and excited-state potential energy surfaces in photochemical modeling, the implication for the simulation of vibrationally resolved optical spectra is minor. Indeed, even the small ASD of $0.02 \mathrm{eV}$ here associated with $\Delta \Delta E_{00}$ calculations corresponds to a spectroscopically significant $\sim 160 \mathrm{~cm}^{-1}$ change in a given vibrational frequency in the excited state relative to the ground state. In these regards, it is more noteworthy that the $\Delta \Delta E_{\mathrm{ad}}$ relaxation energies vary little between methods.

Continuing with the statistics in Table 4 but focusing now on the individual groups of the benchmark set, such an analysis reinforces the above-drawn conclusions from the larger data sets. For example, for each group of organic molecules except group $\mathrm{V}$ (hydrocarbons), the ASDs for $\Delta \Delta E_{\mathrm{ad}}(0.04-0.08 \mathrm{eV})$ and $\Delta \Delta E_{00}(0.02-0.03 \mathrm{eV})$ are markedly smaller than the ASD for $\Delta E_{\mathrm{ve}}(0.35-0.47 \mathrm{eV})$. For group V, the ASD for $\Delta \Delta E_{\mathrm{ad}}$ is $0.20 \mathrm{eV}$; however, from the raw data in Table S5 and the SDs for individual excited states shown in Figure $2 b$, it can be inferred that the relative sizableness of this 
number is mostly due to one single method (M06-HF) differing substantially from all other methods in the description of a single excited state in this group (the $2^{1} \mathrm{~A}$ state of acetylene).

For each group of inorganic molecules, in turn, the calculation of $\Delta \Delta E_{00}$ energy differences is associated with an ASD well below $(0.01-0.04 \mathrm{eV})$ the $0.31-0.38 \mathrm{eV}$ range into which the ASDs for $\Delta E_{\mathrm{ve}}$ fall. A similar observation can be made for the calculation of $\Delta \Delta E_{\text {ad }}$ relaxation energies, although an ASD below $0.1 \mathrm{eV}$ is achieved by group II only. From the raw data in Table S1 and the SDs for individual excited states in Figure 2a, it is found that it is largely because of the results for the $1^{1} \Delta_{\mathrm{u}}, 1^{1} \Pi_{\mathrm{g}}$ and $1^{3} \Pi_{\mathrm{g}}$ states of $\mathrm{N}_{2}$ that the ASD for the homodiatomics of group I reaches $0.21 \mathrm{eV}$. Similarly, scrutiny of Table S3 and Figure 2a reveals that the ASD of $0.15 \mathrm{eV}$ for the small polyatomics of group III derives primarily from very varying results for the $1^{1} \mathrm{~A}^{\prime \prime}$ state of $\mathrm{HCN}$, with $\Delta \Delta E_{\text {ad }}$ estimates ranging from -0.83 (CC2) to $-2.51 \mathrm{eV}$ (M06-HF).

Having already commented on the likely origin of the almost negligible dependence of $\Delta \Delta E_{00}$ on computational method, we now turn to analyzing why also the $\Delta \Delta E_{\text {ad }}$ relaxation energies show relatively small variation between methods. One possible explanation for this result is that the methods tend to provide qualitatively similar descriptions of the character of any given excited state, whereby the effect of excitedstate relaxation is not very different from one method to another. Thus, it is of interest to assess whether the descriptions of the excited states are indeed similar between the methods. Such an assessment would arguably offer a more generic (but less straightforwardly quantifiable) measure of method sensitivity than what an analysis of $\Delta \Delta E_{\text {ad }}$ energies can afford, and was carried out in the following way. 
First, for each covalent bond in each chemical system in the benchmark set, we computed the SD for the estimates of the excited-state bond length obtained by the different methods (denoted $\mathrm{SD}_{\mathrm{ES}}$ ), as well as the $\mathrm{SD}$ for the estimates of the ground/excited-state bond-length difference (denoted $\mathrm{SD}_{\mathrm{FC}}$ ). For the sake of completeness, we also computed the SD for the estimates of the ground-state bond length (denoted $\mathrm{SD}_{\mathrm{GS}}$ ). For the organic molecules, only bonds between heavy atoms were considered. Then, for each system, we computed the average $\mathrm{SD}_{\mathrm{ES}}, \mathrm{SD}_{\mathrm{FC}}$ and $\mathrm{SD}_{\mathrm{GS}}$ values over all bonds, which are denoted $\mathrm{ASD}_{\mathrm{ES}}, \mathrm{ASD}_{\mathrm{FC}}$ and $\mathrm{ASD}_{\mathrm{GS}}$, and are presented in Figures S2a-S2c of the Supporting Information. Finally, the extent to which the methods yield qualitatively similar excited states within a particular group (or within particular groups) of the benchmark set was assessed from the average $\mathrm{ASD}_{\mathrm{ES}}$ and the average $\mathrm{ASD}_{\mathrm{FC}}$ values for the systems in that (those) group (groups). These values are given in Table S10 of the Supporting Information.

From Table $\mathrm{S} 10$, it is noted that particularly the average $\mathrm{ASD}_{\mathrm{ES}}$ and $\mathrm{ASD}_{\mathrm{FC}}$ values for the organic molecules of groups IV-IX are small (0.014 and $0.011 \AA$, respectively). Thus, especially for these molecules, there is a clear tendency of uniformity between the methods in their descriptions of the corresponding excited states, most of which exhibit valence character. This may explain our previous observation that $\Delta \Delta E_{\text {ad }}$ calculations are somewhat less method-dependent for these systems than for the inorganic molecules of groups I-III, whose average $\mathrm{ASD}_{\mathrm{ES}}$ and $\mathrm{ASD}_{\mathrm{FC}}$ values are about a three-fold larger (0.037 and $0.029 \AA$, respectively).

To complement this analysis, for each group of molecules I-IX we also collected from Figures $2 \mathrm{a}-2 \mathrm{c}$ the two systems with the largest and the two systems with the 
smallest SDs for calculated $\Delta \Delta E_{\text {ad }}$ relaxation energies, and then plotted these data against the corresponding $\mathrm{ASD}_{\mathrm{ES}}$ and $\mathrm{ASD}_{\mathrm{FC}}$ values in Figures $\mathrm{S} 3 \mathrm{a}$ and $\mathrm{S} 3 \mathrm{~b}$ of the Supporting Information. Notably, as far as the organic molecules are concerned, these plots corroborate - through linear regression analysis with correlation coefficients of 0.720 and 0.847 - that concurrence between methods on the nature of an excited state tends to reduce the variation in the estimates of $\Delta \Delta E_{\mathrm{ad}}$ for that state. Similarly, since "concurrence" is here quantified geometrically, Figures S3a and S3b help rationalizing why some of the organic compounds exhibit larger SDs for $\Delta \Delta E_{\mathrm{ad}}$ than others - this is largely because of larger variation in the optimized excited-state geometries of these compounds.

Finally, to identify the methods that are the chief contributors to the SDs for $\Delta \Delta E_{\mathrm{ad}}$, we calculated the correlation coefficients between the $\Delta \Delta E_{\mathrm{ad}}$ estimates of each method and, as reference, the corresponding $\Delta \Delta E_{\text {ad }}$ estimates obtained with B3LYP. These results, complemented by an analogous analysis based on $\Delta E_{\mathrm{ve}}$ excitation energies, are presented in Table S11 of the Supporting Information. From a comparison of the $\rho\left(\Delta \Delta E_{\text {ad }}\right)$ values, it can be inferred that M06-HF and CIS are the chief contributors to the variation in $\Delta \Delta E_{\mathrm{ad}}$ estimates for the organic molecules. Furthermore, by observing that the corresponding $\rho\left(\Delta E_{\mathrm{ve}}\right)$ values (that do not depend on excited-state geometries) are considerably larger, it can be concluded that this is because these methods yield excitedstate geometries furthest away from the B3LYP ones. 


\section{3. $\Delta \Delta E_{\text {ad }}$ and $\Delta \Delta E_{00}$ Energy Differences for Different Chemical Systems. Having}

established that the calculation of both $\Delta \Delta E_{\text {ad }}$ and $\Delta \Delta E_{00}$ energy differences is overall surprisingly insensitive to the choice of quantum chemical method, but also observed that this holds true particularly for the latter quantity and for organic molecules, it is further of interest to briefly investigate how these quantities vary between different chemical systems. To this end, Figures $4 \mathrm{a}$ and $4 \mathrm{~b}$ present histograms of calculated $\Delta \Delta E_{\text {ad }}$ and $\Delta \Delta E_{00}$ energy differences for the 96 excited states included in the benchmark set. For each state, the histograms consider the averages of the respective $\Delta \Delta E_{\text {ad }}$ and $\Delta \Delta E_{00}$ values obtained with the BP86, B3LYP, PBE0, M06-2X, M06-HF, CAM-B3LYP, $\omega \mathrm{B} 97 \mathrm{X}-\mathrm{D}, \mathrm{CIS}$ and $\mathrm{CC} 2$ methods. Histograms for the individual methods are shown in Figures S4 and S5 of the Supporting Information.
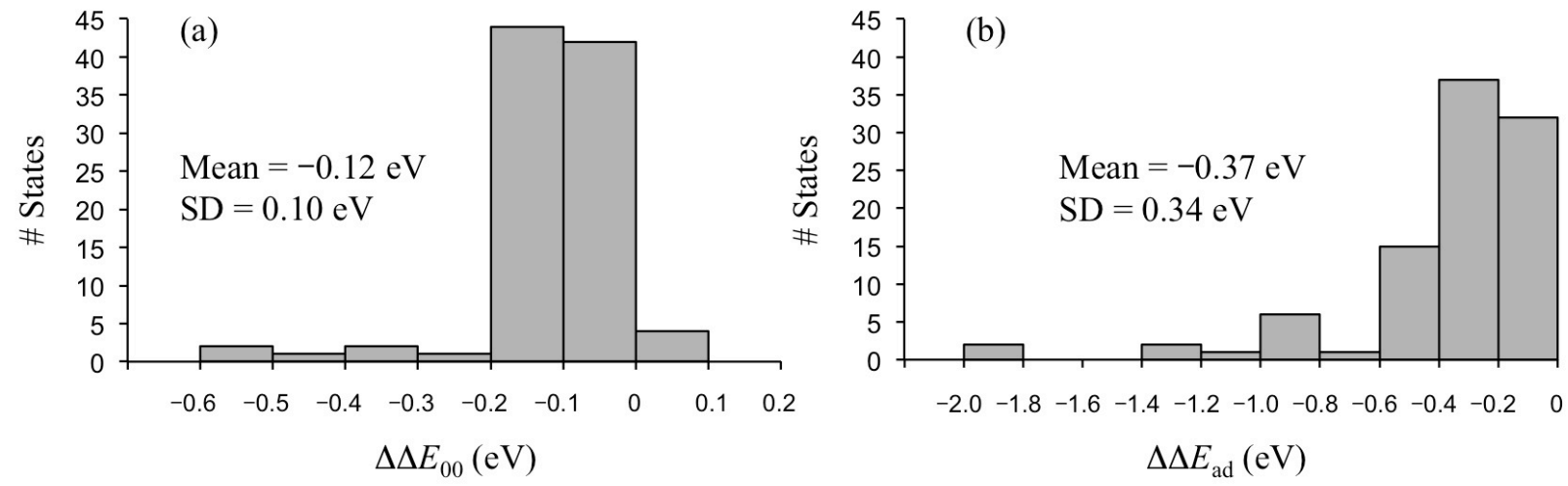

Figure 4. Histograms of calculated $\Delta \Delta E_{00}$ (a) and $\Delta \Delta E_{\text {ad }}$ (b) energy differences for the 96 excited states included in the benchmark set.

Starting with the $\Delta \Delta E_{00}$ histogram in Figure 4a, this quantity varies remarkably little between the different excited states (the SD is only $0.10 \mathrm{eV}$ ), especially considering the breadth of chemical systems included in the benchmark set and their varying sizes 
(from 2 atoms to 46 atoms in terylene). Indeed, of the 96 states, 86 exhibit a $\Delta \Delta E_{00}$ that is negative by up to $0.2 \mathrm{eV}$, whereas only six exhibit a $\Delta \Delta E_{00}$ that is negative by more than $0.2 \mathrm{eV}$. Specifically, $\Delta \Delta E_{00}$ is -0.51 and $-0.55 \mathrm{eV}$ for the $1^{1} \mathrm{~A}^{\prime \prime}{ }_{2}$ and $3^{1} \mathrm{~A}$ states of $\mathrm{NH}_{3}$; 0.43 and $-0.37 \mathrm{eV}$ for the $2^{1} \mathrm{~A}^{\prime}$ and $1^{1} \mathrm{~A}^{\prime \prime}$ states of aminomethane; $-0.38 \mathrm{eV}$ for the $2^{1} \mathrm{~A}^{\prime}$ state of aminoethane; and $-0.21 \mathrm{eV}$ for the $2^{1} \mathrm{~A}$ state of pyridine. Of these states, all but one - the $2^{1} \mathrm{~A}$ state of pyridine - involve the planarization of an amino group when the system relaxes to the excited-state minimum. Altogether, the mean $\Delta \Delta E_{00}$ value for the 96 states is $-0.12 \mathrm{eV}$. Given the small variation in $\Delta \Delta E_{00}$ between the different molecules, this value constitutes a rough but tentatively useful estimate of the magnitude of $\Delta \Delta E_{00}$ for systems too large for explicit calculation of this quantity, provided that the excited state in question does not undergo a substantial geometric rearrangement relative to the ground state. The tendency of $\Delta \Delta E_{00}$ to be negative, i.e., the tendency of ZPVE corrections to lower electronic adiabatic excitation energies by being smaller for the excited state than for the ground state, is a reflection of the fact that excited-state potential energy surfaces are typically flatter than ground-state potential energy surfaces, because of the weakened bonding in the excited state.

Turning to the $\Delta \Delta E_{\text {ad }}$ histogram in Figure $4 \mathrm{~b}$, this quantity, which is always nonpositive and whose mean value for the 96 states is $-0.37 \mathrm{eV}$, varies over a wider range between the different chemical systems (the SD is $0.34 \mathrm{eV})$ than what $\Delta \Delta E_{00}$ does $(0.10$ $\mathrm{eV}$ ). The majority of the states ( 84 of 96 ) show a $\Delta \Delta E_{\text {ad }}$ that is negative by up to $0.6 \mathrm{eV}$. The remaining 12 states whose energies are lowered by more than $0.6 \mathrm{eV}$ upon relaxation from the vertically excited Franck-Condon point include the states mentioned above in which an amino group undergoes a planarization, but also the $1^{1} \Delta_{\mathrm{u}}, 1^{1} \Pi_{\mathrm{g}}$ and $1^{3} \Pi_{\mathrm{g}}$ states 
of $\mathrm{N}_{2}$; the $1^{1} \mathrm{~A}^{\prime \prime}$ state of $\mathrm{HCN}$; the $2^{1} \mathrm{~A}$ states of formic acid and acetylene; and the $1^{1} \mathrm{~B}_{1}$ state of biphenyl.

Finally, from the $\Delta \Delta E_{\mathrm{ad}}$ histograms for the individual methods in Figure S5, we see that similar mean values are obtained for the 96 states, which is a straightforward consequence of the relatively minor dependence of $\Delta \Delta E_{\mathrm{ad}}$ on computational method established in this work. Indeed, the mean values fall within a narrow range between 0.35 and $-0.41 \mathrm{eV}$, with M06-HF $(-0.39 \mathrm{eV})$ and CIS $(-0.41 \mathrm{eV})$ as slight outliers. This is consistent with the results of Table S11.

\section{CONCLUSIONS}

In summary, using seven different density functionals (BP86, B3LYP, PBE0, M06-2X, M06-HF, CAM-B3LYP and wB97X-D) and two ab initio methods (CIS and CC2), we have calculated $\Delta E_{\mathrm{ve}}, \Delta E_{\mathrm{ad}}$ and $\Delta E_{00}$ energies for 96 excited states of 79 different organic and inorganic molecules contained in the benchmark set of $\Delta E_{00}$ energies recently compiled from high-resolution gas-phase experiments by Furche and co-workers. ${ }^{29}$ However, rather than primarily focusing on the accuracy with which the methods considered reproduce the experimental $\Delta E_{00}$ energies of these states, the main motivation for the work is to investigate to what extent the calculation of differences between $\Delta E_{\mathrm{ve}}$, $\Delta E_{\mathrm{ad}}$ and $\Delta E_{00}$ energies is sensitive to the choice of quantum chemical method. Indeed, following a previous observation that - for the chromophores of the photoactive yellow protein and the green fluorescent protein - these differences vary by as little as $\sim 0.1 \mathrm{eV}$ 
between methods, ${ }^{59}$ we believe it is well worthwhile to explore whether a similar trend applies also to a large and variable benchmark set of excited states like the present one.

Through our calculations, a distinct difference in method sensitivity can indeed be inferred between on the one hand $\Delta E_{\mathrm{ve}}$ energies and on the other $\Delta \Delta E_{\mathrm{ad}}=\Delta E_{\mathrm{ad}}-\Delta E_{\mathrm{ve}}$ and $\Delta \Delta E_{00}=\Delta E_{00}-\Delta E_{\text {ad }}$ energy differences. Specifically, while the $\Delta E_{\mathrm{ve}}$ energies obtained with the nine methods exhibit a standard deviation that amounts to $0.39 \mathrm{eV}$ when averaged over all of the 96 excited states, the corresponding standard deviations for $\Delta \Delta E_{\text {ad }}$ and $\Delta \Delta E_{00}$ are only 0.10 and $0.02 \mathrm{eV}$, respectively. Similar statistics are found also when the nine individual groups of molecules in the benchmark set are considered separately, although the average $\Delta E_{\mathrm{ve}}$ and $\Delta \Delta E_{\mathrm{ad}}$ standard deviations are more distinctly different for the six organic groups. Somewhat notably, the average $\Delta \Delta E_{00}$ standard deviation lies within a mere $0.01-0.05 \mathrm{eV}$ for each of the nine groups.

The calculations also enable us to assess the variation in $\Delta \Delta E_{\text {ad }}$ and $\Delta \Delta E_{00}$ between a statistically meaningful number of different chemical systems. From the associated standard deviations and mean values, it is found that the $\Delta \Delta E_{00}$ values tend to reside in a surprisingly narrow range around $-0.12 \mathrm{eV}$. In fact, with the exception of a few systems in which an amino group becomes planar in the excited state, all systems (regardless of chemical features and size) exhibit a $\Delta \Delta E_{00}$ that lies at most $\sim 0.10 \mathrm{eV}$ above or below $-0.12 \mathrm{eV}$. No similar trend applies to the $\Delta \Delta E_{\text {ad }}$ excited-state relaxation energies, which fall within a wider range (in most cases $\pm 0.3 \mathrm{eV}$ ) around the mean value of $-0.37 \mathrm{eV}$.

As for the performance of the different methods in calculating $\Delta E_{00}$ energies, the smallest errors relative to experimental estimates is achieved by $\mathrm{CC} 2$, which shows a 
MAE of $0.19 \mathrm{eV}$ for the full benchmark set, followed by B3LYP, PBE0, CAM-B3LYP and $\omega \mathrm{B} 97 \mathrm{X}-\mathrm{D}$, which afford MAEs in the $0.24-0.27 \mathrm{eV}$ range. For the inorganic systems, the latter methods all show smaller MAEs $(0.21-0.23 \mathrm{eV})$ than CC2 $(0.31 \mathrm{eV})$, whereas $\mathrm{CC} 2$ performs particularly well for the organic systems (MAE of $0.15 \mathrm{eV}$ ) that constitute the bulk of the benchmark set.

Finally, having demonstrated in a quantitative fashion that the task to accurately estimate $\Delta \Delta E_{\text {ad }}$ and $\Delta \Delta E_{00}$ energy differences by means of expensive excited-state geometry optimizations and frequency calculations is less demanding on the quantum chemical level of theory than the task to accurately calculate excitation energies, and also shown that this holds true over a range of possible $\Delta \Delta E_{\text {ad }}$ and $\Delta \Delta E_{00}$ values, we note that a natural continuation of this work and a further contribution to the field of computational photochemistry and photobiology ${ }^{91}$ would be to also consider chromophores in a solvent or protein environment. Such an investigation is a future goal of our research in this field. 


\section{- ASSOCIATED CONTENT}

\section{Supporting Information}

Chemical structures of the molecules in groups IV-IX of the benchmark set are shown in Figure $\mathrm{S} 1 . \mathrm{ASD}_{\mathrm{GS}}, \mathrm{ASD}_{\mathrm{ES}}$ and $\mathrm{ASD}_{\mathrm{FC}}$ values for individual systems in the benchmark set are given in Figures $\mathrm{S} 2 \mathrm{a}-\mathrm{S} 2 \mathrm{c}$. Relationships between SDs for calculated $\Delta \Delta E_{\mathrm{ad}}$ energy differences and $\mathrm{ASD}_{\mathrm{ES}}$ and $\mathrm{ASD}_{\mathrm{FC}}$ values are presented in Figures $\mathrm{S} 3 \mathrm{a}$ and $\mathrm{S} 3 \mathrm{~b}$. Histograms of calculated $\Delta \Delta E_{00}$ and $\Delta \Delta E_{\text {ad }}$ energy differences for the 96 excited states included in the benchmark set at different levels of theory are shown in Figures S4 and S5. Calculated $\Delta E_{\mathrm{ve}}$ energies and $\Delta \Delta E_{\mathrm{ad}}$ and $\Delta \Delta E_{00}$ energy differences for all states in groups I-IX of the benchmark set are given in Tables S1-S9. Average ASD $\mathrm{GS}_{\text {, }} \mathrm{ASD}_{\mathrm{ES}}$ and $\mathrm{ASD}_{\mathrm{FC}}$ values for all states in different groups of the benchmark set are given in Table S10. Correlation coefficients between $\Delta E_{\mathrm{ve}}$ energies and $\Delta \Delta E_{\mathrm{ad}}$ energy differences obtained with B3LYP and all other methods are listed in Table S11. Also provided are a complete author list for reference 82 and atomic coordinates of optimized ground and excited-state geometries. This material is available free of charge via the Internet at http://pubs.acs.org.

\section{- AUTHOR INFORMATION}

\section{Corresponding Author}

*Tel: 46-13-282497. Fax: 46-13-137568. E-mail: bodur@ifm.liu.se.

\section{Notes}

The authors declare no competing financial interest. 


\section{- ACKNOWLEDGMENTS}

This work was supported by Linköping University, the Swedish Research Council, the Olle Engkvist Foundation and the Wenner-Gren Foundations. All calculations were performed at the National Supercomputer Centre (NSC) in Linköping. 


\section{- REFERENCES}

(1) Serrano-Andrés, L.; Merchán, M.; Nebot-Gil, I.; Lindh, R.; Roos, B. O. Towards an Accurate Molecular Orbital Theory for Excited States: Ethene, Butadiene, and Hexatriene. J. Chem. Phys. 1993, 98, 3151-3162. DOI: 10.1063/1.465071

(2) Roos, B. O.; Andersson, K.; Fülscher, M. P.; Malmqvist, P. -Å.; Serrano-Andrés, L.; Pierloot, K.; Merchán, M. Multiconfigurational Perturbation Theory: Applications in Electronic Spectroscopy. In Advances in Chemical Physics: New Methods in Computational Quantum Mechanics; Prigogine, I.; Rice, S. A. (Eds.); Wiley: New York, 1996; Vol. 93; pp 219-331. DOI: 10.1002/9780470141526.ch5

(3) Tozer, D. J.; Amos, R. D.; Handy, N. C.; Roos, B. O.; Serrano-Andrés, L. Does Density Functional Theory Contribute to the Understanding of Excited States of Unsaturated Organic Compounds? Mol. Phys. 1999, 97, 859-868. DOI: 10.1080/ 00268979909482888

(4) Parac, M.; Grimme, S. Comparison of Multireference Møller-Plesset Theory and Time-Dependent Methods for the Calculation of Vertical Excitation Energies of Molecules. J. Phys. Chem. A 2002, 106, 6844-6850. DOI: 10.1021/jp020550e

(5) Grimme, S.; Neese, F. Double-Hybrid Density Functional Theory for Excited Electronic States of Molecules. J. Chem. Phys. 2007, 127, 154116. DOI: 10.1063/ 1.2772854

(6) Peach, M. J. G.; Benfield, P.; Helgaker, T.; Tozer, D. J. Excitation Energies in Density Functional Theory: An Evaluation and a Diagnostic Test. J. Chem. Phys. 2008, 128, 044118. DOI: 10.1063/1.2831900

(7) Schreiber, M.; Silva-Junior, M. R.; Sauer, S. P. A.; Thiel, W. Benchmarks for Electronically Excited States: CASPT2, CC2, CCSD, and CC3. J. Chem. Phys. 2008, 128, 134110. DOI: $10.1063 / 1.2889385$

(8) Silva-Junior, M. R.; Schreiber, M.; Sauer, S. P. A.; Thiel, W. Benchmarks for Electronically Excited States: Time-Dependent Density Functional Theory and Density Functional Theory Based Multireference Configuration Interaction. J. Chem. Phys. 2008, 129, 104103. DOI: 10.1063/1.2973541 
(9) Jacquemin, D.; Perpète, E. A.; Scuseria, G. E.; Ciofini, I.; Adamo, C. TD-DFT Performance for the Visible Absorption Spectra of Organic Dyes: Conventional versus Long-Range Hybrids. J. Chem. Theory Comput. 2008, 4, 123-135. DOI: 10.1021/ ct700187z

(10) Zhao, Y.; Truhlar, D. G. The M06 Suite of Density Functionals for Main Group Thermochemistry, Thermochemical Kinetics, Noncovalent Interactions, Excited States, and Transition Elements: Two New Functionals and Systematic Testing of Four M06Class Functionals and 12 Other Functionals. Theor. Chem. Acc. 2008, 120, 215-241. DOI: $10.1007 / \mathrm{s} 00214-007-0310-\mathrm{X}$

(11) Sauer, S. P. A.; Schreiber, M.; Silva-Junior, M. R.; Thiel, W. Benchmarks for Electronically Excited States: A Comparison of Noniterative and Iterative Triples Corrections in Linear Response Coupled Cluster Methods: CCSDR(3) versus CC3. J. Chem. Theory Comput. 2009, 5, 555-564. DOI: 10.1021/ct800256j

(12) Jacquemin, D.; Wathelet, V.; Perpète, E. A.; Adamo, C. Extensive TD-DFT Benchmark: Singlet-Excited States of Organic Molecules. J. Chem. Theory Comput. 2009, 5, 2420-2435. DOI: $10.1021 /$ ct900298e

(13) Caricato, M.; Trucks, G. W.; Frisch, M. J.; Wiberg, K. B. Electronic Transition Energies: A Study of the Performance of a Large Range of Single Reference Density Functional and Wave Function Methods on Valence and Rydberg States Compared to Experiment. J. Chem. Theory Comput. 2010, 6, 370-383. DOI: 10.1021/ct9005129

(14) Goerigk, L.; Grimme, S. Assessment of TD-DFT Methods and of Various Spin Scaled CIS(D) and CC2 Versions for the Treatment of Low-Lying Valence Excitations of Large Organic Dyes. J. Chem. Phys. 2010, 132, 184103. DOI: 10.1063/1.3418614

(15) Leang, S. S.; Zahariev, F.; Gordon, M. S. Benchmarking the Performance of TimeDependent Density Functional Methods. J. Chem. Phys. 2012, 136, 104101. DOI: $10.1063 / 1.3689445$

(16) Andersson, K.; Malmqvist, P. -Å.; Roos, B. O.; Sadlej, A. J.; Wolinski, K. SecondOrder Perturbation Theory with a CASSCF Reference Function. J. Phys. Chem. 1990, 94, 5483-5488. DOI: 10.1021/j100377a012 
(17) Andersson, K.; Malmqvist, P. -Å.; Roos, B. O. Second-Order Perturbation Theory with a Complete Active Space Self-Consistent Field Reference Function. J. Chem. Phys. 1992, 96, 1218-1226. DOI: 10.1063/1.462209

(18) Stanton, J. F.; Bartlett, R. J. The Equation of Motion Coupled-Cluster Method. A Systematic Biorthogonal Approach to Molecular Excitation Energies, Transition Probabilities, and Excited State Properties. J. Chem. Phys. 1993, 98, 7029-7039. DOI: $10.1063 / 1.464746$

(19) Christiansen, O.; Koch, H.; Jørgensen, P. Response Functions in the CC3 Iterative Triple Excitation Model. J. Chem. Phys. 1995, 103, 7429-7441. DOI: 10.1063/1.470315 (20) Krylov, A. I. Equation-of-Motion Coupled-Cluster Methods for Open-Shell and Electronically Excited Species: The Hitchhiker's Guide to Fock Space. Annu. Rev. Phys. Chem. 2008, 59, 433-462. DOI: 10.1146/annurev.physchem.59.032607.093602

(21) Runge, E.; Gross, E. K. U. Density-Functional Theory for Time-Dependent Systems. Phys. Rev. Lett. 1984, 52, 997-1000. DOI: 10.1103/PhysRevLett.52.997

(22) Bauernschmitt, R.; Ahlrichs, R. Treatment of Electronic Excitations within the Adiabatic Approximation of Time Dependent Density Functional Theory. Chem. Phys. Lett. 1996, 256, 454-464. DOI: 10.1016/0009-2614(96)00440-x

(23) Casida, M. E.; Jamorski, C.; Casida, K. C.; Salahub, D. R. Molecular Excitation Energies to High-Lying Bound States from Time-Dependent Density-Functional Response Theory: Characterization and Correction of the Time-Dependent Local Density Approximation Ionization Threshold. J. Chem. Phys. 1998, 108, 4439-4449. DOI: $10.1063 / 1.475855$

(24) Stratmann, R. E.; Scuseria, G. E.; Frisch, M. J. An Efficient Implementation of Time-Dependent Density-Functional Theory for the Calculation of Excitation Energies of Large Molecules. J. Chem. Phys. 1998, 109, 8218-8224. DOI: 10.1063/1.477483

(25) Marques, M. A. L.; Gross, E. K. U. Time-Dependent Density Functional Theory. Annu. Rev. Phys. Chem. 2004, 55, 427-455. DOI: 10.1146/annurev.physchem.55. 091602.094449

(26) Dreuw, A.; Head-Gordon, M. Single-Reference Ab Initio Methods for the Calculation of Excited States of Large Molecules. Chem. Rev. 2005, 105, 4009-4037. DOI: $10.1021 /$ cr0505627 
(27) Casida, M. E. Time-Dependent Density-Functional Theory for Molecules and Molecular Solids. J. Mol. Struct. (Theochem.) 2009, 914, 3-18. DOI: 10.1016/ j.theochem.2009.08.018

(28) Maitra, N. T.; Zhang, F.; Cave, R. J.; Burke, K. Double Excitations within TimeDependent Density Functional Theory Linear Response. J. Chem. Phys. 2004, 120, 5932-5937. DOI: $10.1063 / 1.1651060$

(29) Send, R.; Kühn, M.; Furche, F. Assessing Excited State Methods by Adiabatic Excitation Energies. J. Chem. Theory Comput. 2011, 7, 2376-2386. DOI: 10.1021/ ct200272b

(30) Christiansen, O.; Koch, H.; Jørgensen, P. The Second-Order Approximate Coupled Cluster Singles and Doubles Model CC2. Chem. Phys. Lett. 1995, 243, 409-418. DOI: 10.1016/0009-2614(95)00841-Q

(31) Schirmer, J. Beyond the Random-Phase Approximation: A New Approximation Scheme for the Polarization Propagator. Phys. Rev. A 1982, 26, 2395-2416. DOI: 10.1103/PhysRevA.26.2395

(32) Schirmer, J.; Trofimov, A. B. Intermediate State Representation Approach to Physical Properties of Electronically Excited Molecules. J. Chem. Phys. 2004, 120, 11449-11464. DOI: $10.1063 / 1.1752875$

(33) Osamura, Y.; Yamaguchi, Y.; Schaefer, III, H. F. Generalization of Analytic Configuration Interaction (CI) Gradient Techniques for Potential Energy Hypersurfaces, Including a Solution to the Coupled Perturbed Hartree-Fock Equations for Multiconfiguration SCF Molecular Wave Functions. J. Chem. Phys. 1982, 77, 383-390.

DOI: $10.1063 / 1.443617$

(34) Foresman, J. B.; Head-Gordon, M.; Pople, J. A.; Frisch, M. J. Toward a Systematic Molecular Orbital Theory for Excited States. J. Phys. Chem. 1992, 96, 135-149. DOI: $10.1021 / \mathrm{j} 100180 \mathrm{a} 030$

(35) Stanton, J. F.; Gauss, J. Analytic Energy Gradients for the Equation-of-Motion Coupled-Cluster Method: Implementation and Application to the HCN/HNC System. $J$. Chem. Phys. 1994, 100, 4695-4698. DOI: 10.1063/1.466253 
(36) Yamamoto, N.; Vreven, T.; Robb, M. A.; Frisch, M. J.; Schlegel, H. B. A Direct Derivative MC-SCF Procedure. Chem. Phys. Lett. 1996, 250, 373-378. DOI: 10.1016/0009-2614(96)00027-9

(37) Nakajima, T.; Nakatsuji, H. Analytical Energy Gradient of the Ground, Excited, Ionized and Electron-Attached States Calculated by the SAC/SAC-CI Method. Chem. Phys. Lett. 1997, 280, 79-84. DOI: 10.1016/s0009-2614(97)01097-x

(38) Gwaltney, S. R.; Bartlett, R. J. Gradients for the Partitioned Equation-of-Motion Coupled-Cluster Method. J. Chem. Phys. 1999, 110, 62-71. DOI: 10.1063/1.478085

(39) Van Caillie, C.; Amos, R. D. Geometric Derivatives of Excitation Energies Using SCF and DFT. Chem. Phys. Lett. 1999, 308, 249-255. DOI: 10.1016/s00092614(99)00646-6

(40) Van Caillie, C.; Amos, R. D. Geometric Derivatives of Density Functional Theory Excitation Energies Using Gradient-Corrected Functionals. Chem. Phys. Lett. 2000, 317, 159-164. DOI: $10.1016 / \mathrm{s} 0009-2614(99) 01346-9$

(41) Furche, F.; Ahlrichs, R. Adiabatic Time-Dependent Density Functional Methods for Excited State Properties. J. Chem. Phys. 2002, 117, 7433-7447. DOI: 10.1063/ 1.1508368

(42) Lischka, H.; Dallos, M.; Shepard, R. Analytic MRCI Gradient for Excited States: Formalism and Application to the $n-\pi^{*}$ Valence- and $n-(3 s, 3 p)$ Rydberg States of Formaldehyde. Mol. Phys. 2002, 100, 1647-1658. DOI: 10.1080/00268970210155121

(43) Köhn, A.; Hättig, C. Analytic Gradients for Excited States in the Coupled-Cluster Model CC2 Employing the Resolution-of-the-Identity Approximation. J. Chem. Phys. 2003, 119, 5021-5036. DOI: 10.1063/1.1597635

(44) Levchenko, S. V.; Wang, T.; Krylov, A. I. Analytic Gradients for the SpinConserving and Spin-Flipping Equation-of-Motion Coupled-Cluster Models with Single and Double Substitutions. J. Chem. Phys. 2005, 122, 224106. DOI: 10.1063/1.1877072 (45) Scalmani, G.; Frisch, M. J.; Mennucci, B.; Tomasi, J.; Cammi, R.; Barone, V. Geometries and Properties of Excited States in the Gas Phase and in Solution: Theory and Application of a Time-Dependent Density Functional Theory Polarizable Continuum Model. J. Chem. Phys. 2006, 124, 094107. DOI: 10.1063/1.2173258 
(46) Caricato, M.; Mennucci, B.; Tomasi, J.; Ingrosso, F.; Cammi, R.; Corni, S.; Scalmani, G. Formation and Relaxation of Excited States in Solution: A New Time Dependent Polarizable Continuum Model Based on Time Dependent Density Functional Theory. J. Chem. Phys. 2006, 124, 124520. DOI: 10.1063/1.2183309

(47) Chiba, M.; Tsuneda, T.; Hirao, K. Excited State Geometry Optimizations by Analytical Energy Gradient of Long-Range Corrected Time-Dependent Density Functional Theory. J. Chem. Phys. 2006, 124, 144106. DOI: 10.1063/1.2186995

(48) Min Rhee, Y.; Casanova, D.; Head-Gordon, M. Performance of Quasi-Degenerate Scaled Opposite Spin Perturbation Corrections to Single Excitation Configuration Interaction for Excited State Structures and Excitation Energies with Application to the Stokes Shift of 9-Methyl-9,10-dihydro-9-silaphenanthrene. J. Phys. Chem. A 2009, 113, 10564-10576. DOI: $10.1021 /$ jp903659u

(49) Dierksen, M.; Grimme, S. Density Functional Calculations of the Vibronic Structure of Electronic Absorption Spectra. J. Chem. Phys. 2004, 120, 3544-3554. DOI: 10.1063/ 1.1642595

(50) Barone, V.; Bloino, J.; Biczysko, M.; Santoro, F. Fully Integrated Approach to Compute Vibrationally Resolved Optical Spectra: From Small Molecules to Macrosystems. J. Chem. Theory Comput. 2009, 5, 540-554. DOI: 10.1021/ct8004744

(51) Kamarchik, E.; Krylov, A. I. Non-Condon Effects in the One- and Two-Photon Absorption Spectra of the Green Fluorescent Protein. J. Phys. Chem. Lett. 2011, 2, 488492. DOI: $10.1021 / \mathrm{jz} 101616 \mathrm{~g}$

(52) Avila Ferrer, F. J.; Cerezo, J.; Stendardo, E.; Improta, R.; Santoro, F. Insights for an Accurate Comparison of Computational Data to Experimental Absorption and Emission Spectra: Beyond the Vertical Transition Approximation. J. Chem. Theory Comput. 2013, 9, 2072-2082. DOI: $10.1021 / \operatorname{ct} 301107 \mathrm{~m}$

(53) Charaf-Eddin, A.; Planchat, A.; Mennucci, B.; Adamo, C.; Jacquemin, D. Choosing a Functional for Computing Absorption and Fluorescence Band Shapes with TD-DFT. $J$. Chem. Theory Comput. 2013, 9, 2749-2760. DOI: 10.1021/ct4000795

(54) Grimme, S.; Izgorodina, E. I. Calculation of 0-0 Excitation Energies of Organic Molecules by CIS(D) Quantum Chemical Methods. Chem. Phys. Lett. 2004, 305, 223230. DOI: $10.1016 /$ j.chemphys.2004.06.050 
(55) Rappoport, D.; Furche, F. Analytical Time-Dependent Density Functional Derivative Methods within the RI-J Approximation, an Approach to Excited States of Large Molecules. J. Chem. Phys. 2005, 122, 064105. DOI: 10.1063/1.1844492

(56) Hellweg, A.; Grün, S. A.; Hättig, C. Benchmarking the Performance of SpinComponent Scaled CC2 in Ground and Electronically Excited States. Phys. Chem. Chem. Phys. 2008, 10, 4119-4127. DOI: 10.1039/b803727b

(57) Jacquemin, D.; Planchat, A.; Adamo, C.; Mennucci, B. TD-DFT Assessment of Functionals for Optical 0-0 Transitions in Solvated Dyes. J. Chem. Theory Comput. 2012, 8, 2359-2372. DOI: $10.1021 / \operatorname{ct} 300326 f$

(58) Winter, N. O. C.; Graf, N. K.; Leutwyler, S.; Hättig, C. Benchmarks for 0-0 Transitions of Aromatic Organic Molecules: DFT/B3LYP, ADC(2), CC2, SOS-CC2 and SCS-CC2 Compared to High-Resolution Gas-Phase Data. Phys. Chem. Chem. Phys. 2013, 15, 6623-6630. DOI: 10.1039/c2cp42694c

(59) Uppsten, M.; Durbeej, B. Quantum Chemical Comparison of Vertical, Adiabatic, and 0-0 Excitation Energies: The PYP and GFP Chromophores. J. Comput. Chem. 2012, 33, 1892-1901. DOI: 10.1002/jcc.23027

(60) Lee, C.; Yang, W.; Parr, R. G. Development of the Colle-Salvetti CorrelationEnergy Formula into a Functional of the Electron Density. Phys. Rev. B 1988, 37, 785789. DOI: $10.1103 /$ PhysRevB.37.785

(61) Becke, A. D. Density-Functional Thermochemistry. III. The Role of Exact Exchange. J. Chem. Phys. 1993, 98, 5648-5652. DOI: 10.1063/1.464913

(62) Stephens, P. J.; Devlin, F. J.; Chabalowski, C. F.; Frisch, M. J. Ab Initio Calculation of Vibrational Absorption and Circular Dichroism Spectra Using Density Functional Force Fields. J. Phys. Chem. 1994, 98, 11623-11627. DOI: 10.1021/j100096a001

(63) Perdew, J. P.; Wang, Y. Accurate and Simple Analytic Representation of the Electron-Gas Correlation Energy. Phys. Rev. B 1992, 45, 13244-13249. DOI: 10.1103/ PhysRevB.45.13244

(64) Perdew, J. P. Density-Functional Approximation for the Correlation Energy of the Inhomogeneous Electron Gas. Phys. Rev. B 1986, 33, 8822-8824. DOI: 10.1103/ PhysRevB.33.8822 
(65) Becke, A. D. Density-Functional Exchange-Energy Approximation with Correct Asymptotic Behavior. Phys. Rev. A 1988, 38, 3098-3100. DOI: 10.1103/PhysRevA. 38.3098

(66) Perdew, J. P.; Burke, K.; Ernzerhof, M. Generalized Gradient Approximation Made Simple. Phys. Rev. Lett. 1996, 77, 3865-3868. DOI: 10.1103/PhysRevLett.77.3865

(67) Tao, J.; Perdew, J. P.; Staroverov, V. N.; Scuseria, G. E. Climbing the Density Functional Ladder: Nonempirical Meta-Generalized Gradient Approximation Designed for Molecules and Solids. Phys. Rev. Lett. 2003, 91, 146401. DOI: 10.1103/PhysRevLett. 91.146401

(68) Adamo, C.; Barone, V. Toward Reliable Density Functional Methods without Adjustable Parameters: The PBE0 Model. J. Chem. Phys. 1999, 110, 6158-6170. DOI: $10.1063 / 1.478522$

(69) Zhao, Y.; Truhlar, D. G. Density Functional for Spectroscopy: No Long-Range SelfInteraction Error, Good Performance for Rydberg and Charge-Transfer States, and Better Performance on Average than B3LYP for Ground States. J. Phys. Chem. A 2006, 110, 13126-13130. DOI: $10.1021 / j p 066479 k$

(70) Yanai, T.; Tew, D. P.; Handy, N. C. A New Hybrid Exchange-Correlation Functional Using the Coulomb-Attenuating Method (CAM-B3LYP). Chem. Phys. Lett. 2004, 393, 51-57. DOI: 10.1016/j.cplett.2004.06.011

(71) Chai, J. -D.; Head-Gordon, M. Systematic Optimization of Long-Range Corrected Hybrid Density Functionals. J. Chem. Phys. 2008, 128, 084106. DOI: 10.1063/ 1.2834918

(72) Chai, J. -D.; Head-Gordon, M. Long-Range Corrected Hybrid Density Functionals with Damped Atom-Atom Dispersion Corrections. Phys. Chem. Chem. Phys. 2008, 10, 6615-6620. DOI: 10.1039/b810189b

(73) Hirata, S.; Head-Gordon, M. Time-Dependent Density Functional Theory for Radicals: An Improved Description of Excited States with Substantial Double Excitation Character. Chem. Phys. Lett. 1999, 302, 375-382. DOI: 10.1016/s0009-2614(99)00137-2 (74) Dunning, T. H. Gaussian Basis Sets for Use in Correlated Molecular Calculations. I. The Atoms Boron through Neon and Hydrogen. J. Chem. Phys. 1989, 90, 1007-1023. DOI: $10.1063 / 1.456153$ 
(75) Weigend, F.; Köhn, A.; Hättig, C. Efficient Use of the Correlation Consistent Basis

Sets in Resolution of the Identity MP2 Calculations. J. Chem. Phys. 2002, 116, 31753183. DOI: $10.1063 / 1.1445115$

(76) Tozer, D. J.; Handy, N. C. Improving Virtual Kohn-Sham Orbitals and Eigenvalues: Application to Excitation Energies and Static Polarizabilities. J. Chem. Phys. 1998, 109, 10180-10189. DOI: $10.1063 / 1.477711$

(77) Hirata, S.; Zhan, C. -G.; Aprà, E.; Windus, T. L.; Dixon, D. A. A New, SelfContained Asymptotic Correction Scheme to Exchange-Correlation Potentials for TimeDependent Density Functional Theory. J. Phys. Chem. A 2003, 107, 10154-10158. DOI: 10.1021/jp035667x

(78) Leininger, T.; Stoll, H.; Werner, H. -J.; Savin, A. Combining Long-Range Configuration Interaction with Short-Range Density Functionals. Chem. Phys. Lett. 1997, 275, 151-160. DOI: 10.1016/s0009-2614(97)00758-6

(79) Tawada, Y.; Tsuneda, T.; Yanagisawa, S.; Yanai, T.; Hirao, K. A Long-RangeCorrected Time-Dependent Density Functional Theory. J. Chem. Phys. 2004, 120, 84258433. DOI: $10.1063 / 1.1688752$

(80) Hättig, C. Geometry Optimizations with the Coupled-Cluster Model CC2 Using the Resolution-of-the-Identity Approximation. J. Chem. Phys. 2003, 118, 7751-7761. DOI: $10.1063 / 1.1564061$

(81) Liu, J.; Liang, W. Z. Analytical Approach for the Excited-State Hessian in TimeDependent Density Functional Theory: Formalism, Implementation, and Performance. $J$. Chem. Phys. 2011, 135, 184111. DOI: 10.1063/1.3659312

(82) Frisch, M. J.; Trucks, G. W.; Schlegel, H. B.; Scuseria, G. E.; Robb, M. A.; Cheeseman, J. R.; Scalmani, G.; Barone, V.; Mennucci, B.; Petersson, G. A.; et al. Gaussian 09, Revision C.01; Gaussian, Inc.: Wallingford CT, 2009.

(83) Ahlrichs, R.; Bär, M.; Häser, M.; Horn, H.; Kölmel, C. Electronic Structure Calculations on Workstation Computers: The Program System TURBOMOLE. Chem. Phys. Lett. 1989, 162, 165-169. DOI: 10.1016/0009-2614(89)85118-8

(84) TURBOMOLE V6.3 2011, a development of University of Karlsruhe and Forschungszentrum Karlsruhe GmbH, 1989-2007, TURBOMOLE GmbH, since 2007; available at: http://www.turbomole.com (accessed February 24, 2014). 
(85) Hättig, C.; Weigend, F. CC2 Excitation Energy Calculations on Large Molecules Using the Resolution of the Identity Approximation. J. Chem. Phys. 2000, 113, 51545161. DOI: $10.1063 / 1.1290013$

(86) Shi, D.; Li, W.; Sun, J.; Zhu, Z. MRCI Study on the Spectroscopic Parameters and Molecular Constants of the $\mathrm{X}^{1} \Sigma^{+}, \mathrm{a}^{3} \Sigma^{+}, \mathrm{A}^{1} \Pi$ and $\mathrm{C}^{1} \Sigma^{-}$Electronic States of the $\mathrm{SiO}$ Molecule. Spectrochim. Acta A 2012, 87, 96-105. DOI: 10.1016/j.saa.2011.11.017

(87) Lee, T. J.; Taylor, P. R. A Diagnostic for Determining the Quality of SingleReference Electron Correlation Methods. Int. J. Quantum Chem. Symp. 1989, 23, 199207. DOI: 10.1002 qua. 560360824

(88) Janssen, C. L.; Nielsen, I. M. B. New Diagnostics for Coupled-Cluster and MøllerPlesset Perturbation Theory. Chem. Phys. Lett. 1998, 290, 423-430. DOI: 10.1016/ s0009-2614(98)00504-1

(89) Nielsen, I. M. B.; Janssen, C. L. Double-Substitution-Based Diagnostics for Coupled-Cluster and Møller-Plesset Perturbation Theory. Chem. Phys. Lett. 1999, 310, 568-576. DOI: 10.1016/s0009-2614(99)00770-8

(90) Falklöf, O.; Durbeej, B. Red-Light Absorption and Fluorescence of Phytochrome Chromophores: A Comparative Theoretical Study. Chem. Phys. 2013, 425, 19-28. DOI: 10.1016/j.chemphys.2013.07.018

(91) El-Khoury, P. Z.; Schapiro, I.; Huntress, M.; Melaccio, F.; Gozem, S.; Frutos, L. M.; Olivucci, M. Computational Photochemistry and Photobiology. In CRC Handbook of Organic Photochemistry and Photobiology, Third Edition; Griesbeck, A.; Oelgemöller, M.; Ghetti, F. (Eds.); CRC Press: Boca Raton, FL, 2012; Vol. 2; pp 1029-1056. DOI: $10.1201 / \mathrm{b} 12252-42$ 


\section{- TABLE OF CONTENTS IMAGE}

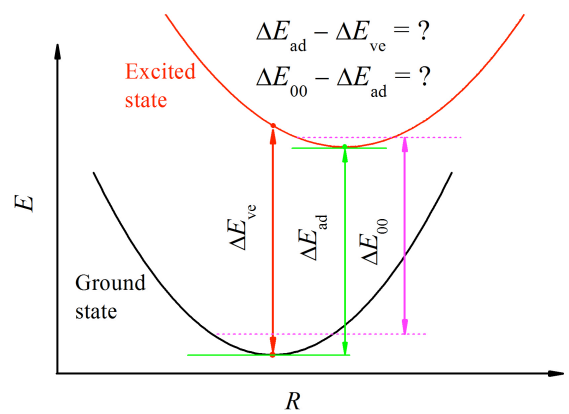

